Research paper

\title{
Impact of renewable energy utilization and artificial intelligence in achieving sustainable development goals
}

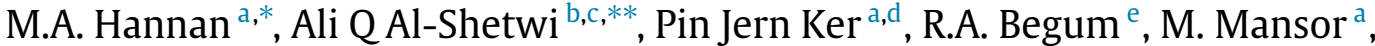 \\ S.A. Rahman ${ }^{\mathrm{a}}$, Z.Y. Dong ${ }^{\mathrm{f}}$, S.K. Tiong ${ }^{\mathrm{d}}$, T.M. Indra Mahlia ${ }^{\mathrm{g}}$, K.M. Muttaqi ${ }^{\mathrm{h}}$ \\ a Department of Electrical Power Engineering, COE, Universiti Tenaga Nasional, Kajang 43000, Malaysia \\ ${ }^{\mathrm{b}}$ Electrical Engineering Department, Fahad Bin Sultan University, Tabuk 71454, Saudi Arabia \\ ${ }^{\mathrm{c}}$ Department of Renewable Energy Engineering, Fahad Bin Sultan University, Tabuk 71454, Saudi Arabia \\ d Institute of Sustainable Energy, Universiti Tenaga Nasional, Kajang 43000, Malaysia \\ e Institute of Climate Change, Universiti Kebangsaan, MalaysiaBangi 43600, Malaysia \\ ${ }^{\mathrm{f}}$ School of Electrical Engineering and Telecommunications, UNSW, Kensington NSW 2033, Australia \\ ${ }^{g}$ School of Information, Systems and Modelling, University of Technology Sydney, Ultimo NSW 2007, Australia \\ ${ }^{\mathrm{h}}$ School of Electrical, Computer and Telecommunications Engineering, University of Wollongong, NSW 2522, Australia
}

\section{A R T I C L E I N F O}

\section{Article history:}

Received 16 July 2021

Received in revised form 13 August 2021

Accepted 24 August 2021

Available online $\mathrm{xxxx}$

\section{Keywords:}

Renewable energy

Energy utilization

Impact analysis

Elicited expert

Artificial intelligence

Sustainable development goals

\begin{abstract}
A B S T R A C T
Many countries around the world are planning to reach $100 \%$ renewable energy use by 2050 . In this context and due to the recent sharp increase in RE utilization in the global energy mix along with its progressive impact on the world energy sector, the evaluation and investigation of its effect on achieving sustainable development goals are not covered sufficiently. Moreover, an assessment of the emerging role of artificial intelligence for renewable energy utilization toward achieving SDGs is conducted. A total of 17 SDGs were divided into three groups, namely, environment, society, and economy, as per the three key pillars of sustainable development. Renewable energy has a positive impact toward achieving 75 targets across all sustainable development goals by using an expert elicitation method-based consensus. However, it may negatively affect the accomplishment of the 27 targets. In addition, artificial intelligence can help renewable energy enable the attainment of 42 out of 169 targets. However, with the current exponential growth of renewable energy share and artificial intelligence development and addressing certain present limitations, this impact may cover additional targets in the future. Nevertheless, recent research foci overlook essential aspects. The exponential growth of renewable energy share and rapid evolution of artificial intelligence need to be accompanied through the requisite regulatory insight and technology regulation to cover additional targets in the future.
\end{abstract}

(C) 2021 The Authors. Published by Elsevier Ltd. This is an open access article under the CC BY-NC-ND license (http://creativecommons.org/licenses/by-nc-nd/4.0/).

\section{Introduction}

Issues concerning the utilization and supply of energy are related to global warming and environmental challenges, such as air pollution, forest destruction, ozone depletion, acid precipitation, greenhouse gases (GHGs), water and land use, wildlife loss, and radioactive emissions (Salim et al., 2018). Besides, the current energy supply impacts the economic and social sectors, thereby causing a range of pressing socio-economic challenges (Simon, 2020). If humanity wants to reach a promising and brighter energy future with reduced environmental, economic, and social

\footnotetext{
* Corresponding author.

** Corresponding author at: Electrical Engineering Department, Fahad Bin Sultan University, Tabuk 71454, Saudi Arabia.

E-mail addresses: hannan@uniten.edu.my (M.A. Hannan), aalshetwi@fbsu.edu.sa (A.Q. Al-Shetwi).
}

impacts, these challenges need to be addressed together. For this purpose and to overcome the well-known adverse effects of conventional energy plants (i.e., coal, oil, and natural gas) on sustainability development, the world now turned to use these resources within some limit. It turned the thinking toward renewable energy (RE) sources (i.e., wind, solar photovoltaic (PV), hydropower, geothermal, tidal, biomass, etc.) (Ediger, 2019). With the use of RE sources, the environmental, economic, and social issues can be reduced because these options are considered to help achieve an environmentally sound technology; low electricity cost; job creation; improved health; community development, especially in rural areas and developing countries; no or little emission production of poisonous and exhaust gases, such as sulfur dioxide, carbon monoxide, and carbon dioxide (Kumar, 2020a). Therefore, by the end of 2020, the renewable power sector has reached an all-time high of more than $256 \mathrm{GW}$ of added capacity. Worldwide, the total installed renewable power 
capacity grew almost 10\% to reach 2839 GW (Global Status Report, 2021). The electricity generation capacity via renewable sources in 2020 has be increased by $139 \mathrm{GW}$ of solar PV, $93 \mathrm{GW}$ of installed wind, $20 \mathrm{GW}$ of hydropower, $0.1 \mathrm{GW}$ of new geothermal power generating capacity came online in 2020, bringing the global total to around $14.1 \mathrm{GW}$, and $527 \mathrm{MW}$ of ocean power including tidal capacity (Global Status Report, 2021). However, based on the International Energy Agency (IEA) (International Energy Agency (IEA), 2021), RE use increased 3\% in 2020 as demand for all other fuels declined. The primary driver was an almost $7 \%$ growth in electricity generation from renewable sources. Accordingly, the share of renewables in global electricity generation jumped to $29 \%$ in 2020, up from 27\% in 2019 . Bioenergy use in industry grew $3 \%$, but was largely offset by a decline in biofuels as lower oil demand also reduced the use of blended biofuels. Renewable electricity generation in 2021 is set to expand by more than $8 \%$, the fastest year-on-year growth since the 1970s. Solar PV and wind are set to contribute two-thirds of renewables growth. Increases in electricity generation from all renewable sources should push the share of renewables in the electricity generation mix to an all-time high of $30 \%$ in 2021 (International Energy Agency (IEA), 2021). In the same context, after years of development and evolution, artificial intelligence (AI) now began to affect our daily lives deeply and is starting to have a considerable impact on the fields of sustainable growth and development (Vinuesa et al., 2020). In line with this, AI has an essential role in the dramatic increase in RE utilization and contribution to the energy mix, as well as the potential to revolutionize the RE sector (Vinuesa et al., 2020; Jha et al., 2017). The AI applications and approaches for developing the RE include safety and reliability improvement, cost reduction, strategies to reduce environmental and climate impacts, increase the energy efficiency, expand RE market, improve integration of microgrids (MG) and smart grid, produce more accurate predictions of RE, and optimal operation of RE sources (Zahraee et al., 2016). Other methods have been used to improve the tidal energy turbine for better performance and efficiency (Alipour et al., 2020a,b). Therefore, based on the literature studies, the applications of AI for RE operation and utilization (AI-based RE) always had a positive impact on RE utilization, and the negative impact is almost negligible.

The RE utilization and AI-based RE have a role in the future of sustainability, either for long- or short-term. Based on the literature, the potential effects of RE and AI-based RE positively affect sustainable development, while the RE utilization may have some negative impacts (Vinuesa et al., 2020; Buonocore et al., 2019; Nerini et al., 2018). Some recent studies focused on linking the relationship between the achievement of the 7th goal (affordable and clean energy) of the sustainable development goals (SDGs) and RE (Ediger, 2019; Swain and Karimu, 2020). However, no research article has systematically examined the degree to which RE utilization and AI-based-RE could affect all facets of sustainable development identified in this research. The 169 targets of the 17 SDGs agreed internationally in the sustainable development agenda 2030 (GA, 2015). This research gap is critical. Thus, it must be filled due to the fact that the RE utilization could affect the capacity to achieve the 169 targets of SDGs.

Therefore, this study presents, discusses, and explores how RE can either affect the achievement of all 169 targets within the 17 goals which are defined in the sustainable development agenda 2030 positively or negatively. Moreover, if the RE impacts a specific target, we discussed and identified if $\mathrm{AI}$ implementation has a role on RE for this particular target. Connections were defined by the processes outlined in the Method section below. The method can be characterized by an expert elicitation methodbased consensus which was notified by earlier research targeted at mapping SDGs interlinkages (Nerini et al., 2018, 2019; DeNeve and Sachs, 2020). Moreover, we present an assessment attempt based on the published evidence, which is characterized as an enabler or inhibitor to identify the impact of RE utilization and development on the pursuit of the 17 SDGs and every one of its constituent targets. The evidence on the impact of AI utilization in RE is also investigated. The RE sources considered in this study include wind, solar PV, hydropower, tidal, marine, geothermal, and biomass energies. Concerning AI utilization for RE sources, although the definition of $\mathrm{AI}$ is not internationally accepted, each software technology that has at least one of the following features was considered AI for this study: optimization (i.e., optimal operation of RE sources and efficiency enhancement), prediction (i.e., weather forecast), interactive communication (i.e., smart grids), automatic knowledge extraction (i.e., scheduling based on the price and whether conduction), and efficient problems solving (i.e., reduce cost and $\mathrm{CO}_{2}$, modeling, availability prediction, and control of RE systems). Other sub-files, such as machine learning, are considered AI, and its impact on RE systems is also considered.

\section{Methods of the study}

This section describes the procedure used to achieve the findings reported in the current. The main aims of this study were to discuss and explore how RE can either affect the achievement of the adopted 2030 agenda positively or negatively for sustainable development that includes 17 SDGs with their 169 targets. Moreover, if the RE has an impact on a specific target, then we discussed and identified the role of the AI implementation on RE in this particular target. Toward this goal, we performed an expert elicitation procedure focused on consensus, advised by previous research on the interlinkage mapping of SDGs. The writers of this research are part of the academic field that covers a wide assortment of areas, especially concerning energy, engineering, environmental, and natural sciences, which serve as experts within the elicitation technique. To support the established linkage between RE and AI utilization for RE with the 169 targets, the authors conducted an expert-driven academic publication search. In this search, the following sources of knowledge were deemed to be appropriate evidence: $(a)$ research published on actual-international applications provided that the analyzed publications have adequate quality; $(b)$ research published on laboratory/controlled eventualities provided that the publications taken into consideration within the evaluation have been of adequate quality; (c) published data, reports, and published evidence from authorized/accredited organizations, such as government bodies and UN; and $(d)$ applications recorded on the commercial level. The following resources of knowledge were not deemed to be sufficient proof: $(a)$ actual-international applications with lack of peer-reviewed study; $(b)$ media; $(c)$ public beliefs; $(d)$ educated assumptions; and $(e)$ other means of knowledge.

\subsection{Expert elicitation process}

In this stage, one or more key contributors were assigned to particular SDGs. However, in certain cases, the same SDG was assigned to more of other contributors. To this end, the first search was carried out by the first contributor, and the additional contributor added the analysis. In some cases, the first contributors added the analysis along with the additional one. The authors have made an effort to search for each target and provided evidence that has adequate quality, if available. When the assessment is finished for each SDG, a reviewer/s evaluated the contact and causes. The reviewers' key contribution is to assess the study objectively and provide alternate points of view. Finally, a broad discussion for the outcomes with respect to 


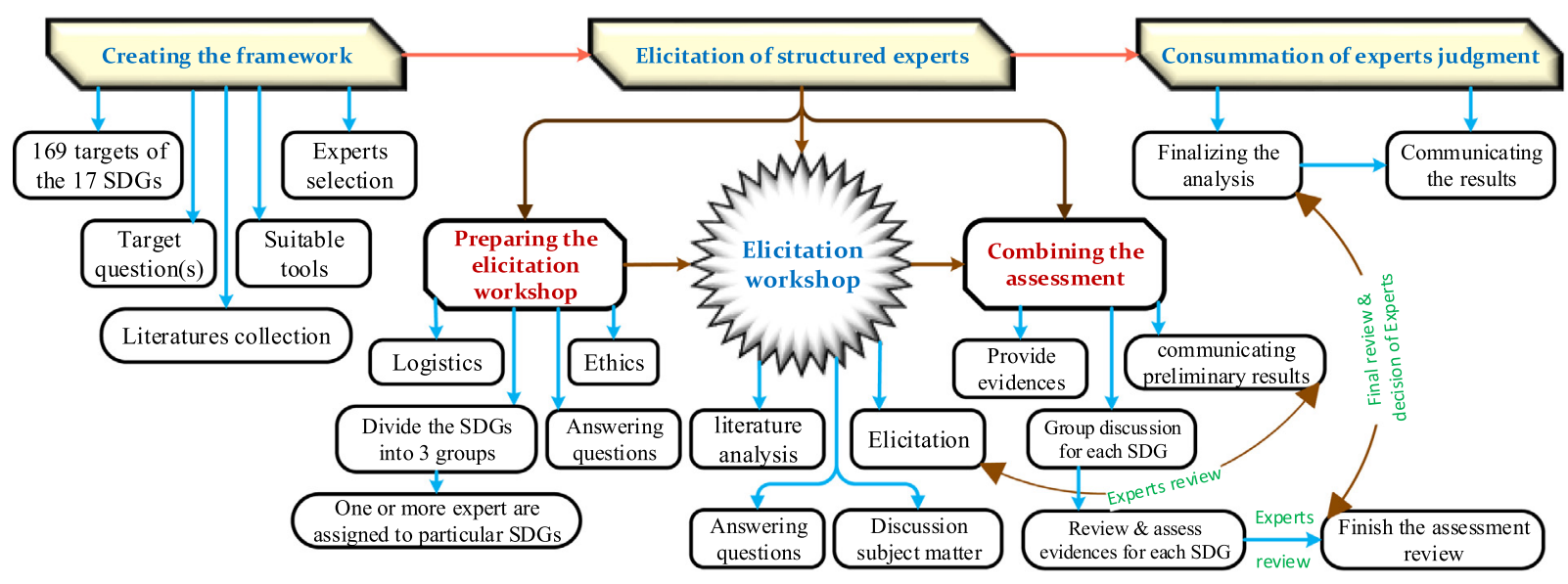

Fig. 1. Expert elicitation method based consensus for the impacts the achievement of all SDGs.

every single goal between the contributors and reviewers was carried out recursively until the assessment for the 17 goals was fairly perfect. The detailed expert elicitation assessment is done based on creating a framework, elicitation of expert structure, and consumption of experts' judgment, as illustrated in Fig. 1.

\subsection{Final analysis}

In this stage, after finding consensus on the assessment of the evidence for each goal (briefly shown in Table 1), analysis of the final results has been conducted by determining the number of targets for which RE utilization acts positively, negatively, or if no evidence is found. The final assessment in percentage is shown in Table 2. Then, when the RE has a positive impact on a specific target, the following question is answered based on the published evidence "Is there any role for AI application for helping the RE to serve as an enabler for this target?" and the number of targets in this regard is evaluated. Next, we evaluated the findings and measured the proportion (in percentage \%) as a positive impact, negative impact, $\mathrm{AI}$ role, and no effects for every one of the 169 targets within the 17 goals (Fig. 2 based on the final results of Table 1). The 17 SDGs were divided into three groups, including Environment, Society, and economy, following the grouping/classifications described by Ref. Giddings et al. (2002) and Savelyeva and Douglas (2017). Observing the individual outcomes from each of these groups is highlighted. For each goal of each SDG, these statistics show whether any reported evidence has a positive or negative effect, and the positive role of AI application in RE utilization was identified. A summary of the findings can be found in Fig. 2.

\subsection{Limitations of the research}

The investigation and assessment presented in this study reflect the authors' point of view. In addition, a few works of literature on how the RE and role of AI applications for RE utilization influence certain targets within the 17 SDGs could have been overlooked by the contributors, or proof of such interlinkage could not be reported yet. However, the approaches were used to reduce the assessment's subjectivity. For every interlinkage, several authors assessed, analyzed, and reviewed various published evidence on the impact of RE utilization and AI applications on RE sources carefully to highlight its delivery of every target, as discussed in the section of the expert elicitation process above. In this regard, it is important to mention that the experts who are fully specialized in social sciences are not present on the board - which can be seen as a shortcoming. Apart from that, and based on the literature, the applications of AI for RE operation and utilization (AI-based RE) always had a positive impact on improving RE utilization, and the negative impact is almost negligible. Therefore, even though the negative impacts in this regard are insignificant, not taking this option in the assessment provided could be considered a limitation in this study. One more limitation is that the RE used in this analysis is limited to the power generation resources, such as PV, wind, geothermal, biomass, hydropower, and marine energies. Lastly, this research is based on SDGs analysis. The SDGs offer a solid perspective to look at globally defined objectives on sustainable development and provide a path forward for reflecting all aspects of sustainability, including social sustainability, human rights (United Nations Human Rights, 2016), environmental development, and economic outcomes. However, the SDGs are a political settlement and could be restricted to describing a few dynamic complexities and cross-interactions among different targets. Consequently, it must be considered as a complement along with former, existing and other international norms in place of a replacement (Nerini et al., 2019).

\section{Assessment on the impacts of RE utilization toward achiev- ing SDGs}

To assess the impact of RE and AI-based RE utilization on each of the 169 targets within the 17 SDGs in the 2030 agenda, we undertook a method designed to answer two questions: (i) Are pieces of evidence of the RE utilization role either positively or negatively toward achieving this particular target published? If yes, then the second question is (ii) Are pieces of evidence about the role of the implementation of AI to the RE systems toward achieving this specific target available? The review assessment of the related literature indicates that RE has a positive impact on 75 targets, which represent $44.3 \%$ across the entire SDGs. However, with the current exponential growth of RE share, these impacts may cover additional targets in the future. Furthermore, 27 targets $(15.8 \%$, across the whole of SDGs) can reveal the negative effect of the blooming of RE. Also, based on the literature (Vinuesa et al., 2020; Jha et al., 2017; Hannan et al., 2020e) and the review of relevant evidence, AI positively impacts RE utilization, such as improving efficiency, operation, and production while reducing the cost and emissions. Thus, AI use in RE has a positive role and may act as an enabler toward achieving 42 targets (24.85\% of all SDGs), commonly by technical enhancement, which can allow these existing limitations to be overcome. To organize the outcomes of this review, the SDGs are classified into three groups as per the three key pillars of sustainable development, particularly 
Table 1

Evidence of RE including AI on the achievement of all 169 targets within the 17 goals.

\begin{tabular}{|c|c|c|c|c|c|c|c|c|c|c|c|}
\hline $\begin{array}{l}\text { Tar- } \\
\text { get }\end{array}$ & Impact & Ref. & $\begin{array}{l}\text { Tar- } \\
\text { get }\end{array}$ & Impact & Ref. & Target & Impact & Ref. & Target & Impact & Ref. \\
\hline $\begin{array}{l}\text { SDG } \\
1.1\end{array}$ & $\mathrm{P}$ & $\begin{array}{l}\text { Nissing and Von Blottnitz } \\
\text { (2010), Sharma (2018) and } \\
\text { Cunha et al. (2021) }\end{array}$ & $\begin{array}{l}\text { SDG } \\
5.6\end{array}$ & $\mathrm{~N} / \mathrm{A}$ & - & $\begin{array}{l}\text { SDG } \\
10.7\end{array}$ & $\mathrm{~N} / \mathrm{A}$ & - & $\begin{array}{l}\text { SDG } \\
15.4\end{array}$ & $\mathrm{~N} / \mathrm{A}$ & - \\
\hline $\begin{array}{l}\text { SDG } \\
1.2\end{array}$ & $P \& N$ & $\begin{array}{l}\text { Zahnd and Kimber (2009) } \\
\text { and Diachuk et al. (2018) }\end{array}$ & $\begin{array}{l}\text { SDG } \\
5.7\end{array}$ & $\mathrm{~N} / \mathrm{A}$ & - & $\begin{array}{l}\text { SDG } \\
10.8\end{array}$ & $\mathrm{~N} / \mathrm{A}$ & - & $\begin{array}{l}\text { SDG } \\
15.5\end{array}$ & $\mathrm{~N} / \mathrm{A}$ & - \\
\hline $\begin{array}{l}\text { SDG } \\
1.3\end{array}$ & $\mathrm{~N} / \mathrm{A}$ & - & $\begin{array}{l}\text { SDG } \\
5.8\end{array}$ & $\mathrm{~N} / \mathrm{A}$ & - & $\begin{array}{l}\text { SDG } \\
10.9\end{array}$ & $\mathrm{~N} / \mathrm{A}$ & - & $\begin{array}{l}\text { SDG } \\
15.6\end{array}$ & $\mathrm{~N} / \mathrm{A}$ & - \\
\hline $\begin{array}{l}\text { SDG } \\
1.4\end{array}$ & $P \& N$ & $\begin{array}{l}\text { Ayodele et al. (2019), Ding } \\
\text { et al. (2019) and Nelson and } \\
\text { Kuriakose (2017) }\end{array}$ & $\begin{array}{l}\text { SDG } \\
5.9\end{array}$ & $\mathrm{~N} / \mathrm{A}$ & - & $\begin{array}{l}\text { SDG } \\
10.10\end{array}$ & $\mathrm{~N} / \mathrm{A}$ & - & $\begin{array}{l}\text { SDG } \\
15.7\end{array}$ & $\mathrm{~N} / \mathrm{A}$ & - \\
\hline $\begin{array}{l}\text { SDG } \\
1.5\end{array}$ & $P$ & $\begin{array}{l}\text { Al-Waeli et al. (2017), } \\
\text { Okkonen and Lehtonen } \\
\text { (2016) and Hussein (2017) }\end{array}$ & $\begin{array}{l}\text { SDG } \\
6.1\end{array}$ & $\begin{array}{l}\text { P \& } \\
P(A I)\end{array}$ & $\begin{array}{l}\text { Phuangpornpitak and } \\
\text { Katejanekarn (2016), Halder } \\
\text { et al. (2016), Keith and } \\
\text { French (2019) and Li et al. } \\
\text { (2018) }\end{array}$ & $\begin{array}{l}\text { SDG } \\
11.1\end{array}$ & $\begin{array}{l}\mathrm{P}, \mathrm{N}, \\
P(A I)\end{array}$ & $\begin{array}{l}\text { Sovacool (2013) and } \\
\text { Situmorang et al. (2020) }\end{array}$ & $\begin{array}{l}\text { SDG } \\
15.8\end{array}$ & $P \& N$ & $\begin{array}{l}\text { Poggi et al. (2018), Bukhary } \\
\text { et al. (2018) and Semeraro } \\
\text { et al. (2018) }\end{array}$ \\
\hline $\begin{array}{l}\text { SDG } \\
1.6\end{array}$ & $P$ & $\begin{array}{l}\text { Schwerhoff and Sy (2017) } \\
\text { and Cloke et al. (2017) }\end{array}$ & $\begin{array}{l}\text { SDG } \\
6.2\end{array}$ & $\begin{array}{l}\text { P \& } \\
P(A I)\end{array}$ & $\begin{array}{l}\text { Gontijo et al. (2018) and } \\
\text { Goel and Sharma (2016) }\end{array}$ & $\begin{array}{l}\text { SDG } \\
11.2\end{array}$ & $\begin{array}{l}\text { P \& } \\
P(A I)\end{array}$ & $\begin{array}{l}\text { Shaukat et al. (2018) and } \\
\text { Manikandan et al. (2018) }\end{array}$ & $\begin{array}{l}\text { SDG } \\
15.9\end{array}$ & $\begin{array}{l}\mathrm{P}, \mathrm{N}, \\
P(A I)\end{array}$ & $\begin{array}{l}\text { Gasparatos et al. (2017), } \\
\text { Gove et al. (2016) and } \\
\text { Dhunny et al. (2019) }\end{array}$ \\
\hline $\begin{array}{l}\text { SDG } \\
1.7\end{array}$ & $\mathrm{~N} / \mathrm{A}$ & - & $\begin{array}{l}\text { SDG } \\
6.3\end{array}$ & $\begin{array}{l}\text { P \& } \\
P(A I)\end{array}$ & $\begin{array}{l}\text { Phuangpornpitak and } \\
\text { Katejanekarn (2016), Halder } \\
\text { et al. (2016), Keith and } \\
\text { French (2019) and Li et al. } \\
\text { (2018) }\end{array}$ & $\begin{array}{l}\text { SDG } \\
11.3\end{array}$ & $\mathrm{~N} / \mathrm{A}$ & - & $\begin{array}{l}\text { SDG } \\
15.10\end{array}$ & $\mathrm{~N} / \mathrm{A}$ & - \\
\hline $\begin{array}{l}\text { SDG } \\
2.1\end{array}$ & $\begin{array}{l}\text { P \& } \\
P(A I)\end{array}$ & $\begin{array}{l}\text { Bryant et al. (2010) and } \\
\text { Behera et al. (2019) }\end{array}$ & $\begin{array}{l}\text { SDG } \\
6.4\end{array}$ & $\begin{array}{l}\text { P \& } \\
P(A I)\end{array}$ & $\begin{array}{l}\text { Hassanien et al. (2016), } \\
\text { Barron-Gafford et al. (2019), } \\
\text { Bertsiou et al. (2018) and } \\
\text { Chaouali et al. (2018) }\end{array}$ & $\begin{array}{l}\text { SDG } \\
11.4\end{array}$ & $\mathrm{~N} / \mathrm{A}$ & - & $\begin{array}{l}\text { SDG } \\
15.11\end{array}$ & $\mathrm{~N}$ & $\begin{array}{l}\text { Gasparatos et al. (2017), } \\
\text { Schuster et al. (2015) and } \\
\text { Frid et al. (2012) }\end{array}$ \\
\hline $\begin{array}{l}\text { SDG } \\
2.2\end{array}$ & $\mathrm{~N} / \mathrm{A}$ & - & $\begin{array}{l}\text { SDG } \\
6.5\end{array}$ & $\mathrm{~N} / \mathrm{A}$ & - & $\begin{array}{l}\text { SDG } \\
11.5\end{array}$ & $\begin{array}{l}\text { P \& } \\
P(A I)\end{array}$ & $\begin{array}{l}\text { Organization WH (2014), } \\
\text { Heidari and Pearce (2016) } \\
\text { and Grabara et al. (2021) }\end{array}$ & $\begin{array}{l}\text { SDG } \\
15.12\end{array}$ & $\mathrm{~N} / \mathrm{A}$ & - \\
\hline $\begin{array}{l}\text { SDG } \\
2.3\end{array}$ & $\begin{array}{l}\text { P \& } \\
P(A I)\end{array}$ & $\begin{array}{l}\text { Appel et al. (2016), } \\
\text { Carroquino et al. (2015) and } \\
\text { Elkadeem et al. (2019) }\end{array}$ & $\begin{array}{l}\text { SDG } \\
6.6\end{array}$ & $\mathrm{~N}$ & $\begin{array}{l}\text { Gasparatos et al. (2017) and } \\
\text { Drechsler et al. (2017a) }\end{array}$ & $\begin{array}{l}\text { SDG } \\
11.6\end{array}$ & $\begin{array}{l}\text { P \& } \\
P(A I)\end{array}$ & $\begin{array}{l}\text { Nachrowi (2012), Zhu et al. } \\
\text { (2020) and Abdolrasol et al. } \\
\text { (2018) }\end{array}$ & & & \\
\hline $\begin{array}{l}\text { SDG } \\
2.4\end{array}$ & $\mathrm{~N} / \mathrm{A}$ & - & $\begin{array}{l}\text { SDG } \\
6.7\end{array}$ & $\mathrm{~N} / \mathrm{A}$ & - & $\begin{array}{l}\text { SDG } \\
11.7\end{array}$ & $\mathrm{~N} / \mathrm{A}$ & - & $\begin{array}{l}\text { SDG } \\
16.1\end{array}$ & $\mathrm{~N} / \mathrm{A}$ & - \\
\hline $\begin{array}{l}\text { SDG } \\
2.5\end{array}$ & $\mathrm{~N} / \mathrm{A}$ & - & $\begin{array}{l}\text { SDG } \\
6.8\end{array}$ & $\mathrm{~N} / \mathrm{A}$ & - & $\begin{array}{l}\text { SDG } \\
11.8\end{array}$ & $\begin{array}{l}\mathrm{P}, \mathrm{N}, \\
P(A I)\end{array}$ & $\begin{array}{l}\text { Kumar (2020b), Ramakumar } \\
\text { and Hughes (1981) and } \\
\text { Owusu and } \\
\text { Asumadu-Sarkodie (2016) }\end{array}$ & $\begin{array}{l}\text { SDG } \\
16.2\end{array}$ & $P \& N$ & $\begin{array}{l}\text { Zahnd and Kimber (2009) } \\
\text { and Thiam D.R. } \\
\text { Renewable energy (2011) }\end{array}$ \\
\hline $\begin{array}{l}\text { SDG } \\
2.6\end{array}$ & $\mathrm{~N} / \mathrm{A}$ & - & $\begin{array}{l}\text { SDG } \\
7.1\end{array}$ & $\begin{array}{l}\mathrm{P} \& \\
P(A I)\end{array}$ & $\begin{array}{l}\text { Abdolrasol et al. (2018), } \\
\text { Wang et al. (2020), Adefarati } \\
\text { and Bansal R.C. Reliability } \\
\text { (2019), Kharchenko et al. } \\
\text { (2019), Yang et al. (2019), } \\
\text { Jones (2018) and Boza and } \\
\text { Evgeniou (2021) }\end{array}$ & $\begin{array}{l}\text { SDG } \\
11.9\end{array}$ & $\mathrm{~N} / \mathrm{A}$ & - & $\begin{array}{l}\text { SDG } \\
16.3\end{array}$ & $\mathrm{~N} / \mathrm{A}$ & - \\
\hline $\begin{array}{l}\text { SDG } \\
2.7\end{array}$ & $\mathrm{~N} / \mathrm{A}$ & - & $\begin{array}{l}\text { SDG } \\
7.2\end{array}$ & $\begin{array}{l}\mathrm{P} \& \\
P(A I)\end{array}$ & $\begin{array}{l}\text { Global Status Report (2021), } \\
\text { Jones (2018), Bowen et al. } \\
\text { (2020), Al-Shetwi et al. } \\
(2020 \text { b) and Ramchurn et al. } \\
(2012)\end{array}$ & $\begin{array}{l}\text { SDG } \\
11.10\end{array}$ & $\begin{array}{l}\text { P \& } \\
P(A I)\end{array}$ & $\begin{array}{l}\text { Callithen and Matthew } \\
\text { (2007), Kammen and Sunter } \\
(2016) \text { and Le Guen et al. } \\
(2018)\end{array}$ & $\begin{array}{l}\text { SDG } \\
16.4\end{array}$ & $\mathrm{~N} / \mathrm{A}$ & - \\
\hline $\begin{array}{l}\text { SDG } \\
2.8\end{array}$ & $\mathrm{P}$ & $\begin{array}{l}\text { Chel and Kaushik (2011) and } \\
\text { Omri and Belaïd (2021) }\end{array}$ & $\begin{array}{l}\text { SDG } \\
7.3\end{array}$ & $\begin{array}{l}\mathrm{P} \& \\
P(A I)\end{array}$ & $\begin{array}{l}\text { Gielen et al. (2019), Gökgöz } \\
\text { and Güvercin (2018) and } \\
\text { Herweijer and Waughray } \\
\text { (2018) }\end{array}$ & $\begin{array}{l}\text { SDG } \\
12.1\end{array}$ & $\mathrm{~N} / \mathrm{A}$ & - & $\begin{array}{l}\text { SDG } \\
16.5\end{array}$ & $\mathrm{~N} / \mathrm{A}$ & - \\
\hline $\begin{array}{l}\text { SDG } \\
3.1\end{array}$ & $\mathrm{~N} / \mathrm{A}$ & - & $\begin{array}{l}\text { SDG } \\
7.4\end{array}$ & $\begin{array}{l}\text { P \& } \\
P(A I)\end{array}$ & $\begin{array}{l}\text { Boza and Evgeniou (2021), } \\
\text { Chandel et al. (2016), } \\
\text { Batmunkh et al. (2018) and } \\
\text { Bagheri et al. (2018) }\end{array}$ & $\begin{array}{l}\text { SDG } \\
12.2\end{array}$ & $\begin{array}{l}\mathrm{P} \& \\
P(A I)\end{array}$ & $\begin{array}{l}\text { Banos et al. (2011) and } \\
\text { Olatomiwa et al. (2016) }\end{array}$ & $\begin{array}{l}\text { SDG } \\
16.6\end{array}$ & $\mathrm{~N} / \mathrm{A}$ & - \\
\hline $\begin{array}{l}\text { SDG } \\
3.2\end{array}$ & $\mathrm{~N} / \mathrm{A}$ & - & $\begin{array}{l}\text { SDG } \\
7.5\end{array}$ & $\begin{array}{l}\mathrm{P} \& \\
P(A I)\end{array}$ & $\begin{array}{l}\text { Boza and Evgeniou (2021), } \\
\text { Philibert (2017), Hu et al. } \\
\text { (2018) and Chen et al. } \\
\text { (2021) }\end{array}$ & $\begin{array}{l}\text { SDG } \\
12.3\end{array}$ & $\mathrm{~N} / \mathrm{A}$ & - & $\begin{array}{l}\text { SDG } \\
16.7\end{array}$ & $\mathrm{~N} / \mathrm{A}$ & - \\
\hline $\begin{array}{l}\text { SDG } \\
3.3\end{array}$ & $\mathrm{~N} / \mathrm{A}$ & - & $\begin{array}{l}\text { SDG } \\
8.1\end{array}$ & $\mathrm{P}$ & $\begin{array}{l}\text { Grabara et al. (2021), Saidi } \\
\text { and Mbarek (2016) and Shen } \\
\text { et al. (2020) }\end{array}$ & $\begin{array}{l}\text { SDG } \\
12.4\end{array}$ & $\begin{array}{l}\mathrm{P}, \mathrm{N}, \\
P(A I)\end{array}$ & $\begin{array}{l}\text { Cremiato et al. (2018), } \\
\text { Münster and Meibom (2011) } \\
\text { and Korai et al. (2016) }\end{array}$ & $\begin{array}{l}\text { SDG } \\
16.8\end{array}$ & $\mathrm{~N} / \mathrm{A}$ & - \\
\hline $\begin{array}{l}\text { SDG } \\
3.4\end{array}$ & $\mathrm{P}$ & $\begin{array}{l}\text { Olatomiwa et al. (2018) and } \\
\text { Al-faruq et al. (2016) }\end{array}$ & $\begin{array}{l}\text { SDG } \\
8.2\end{array}$ & $\begin{array}{l}\mathrm{P}, \mathrm{N}, \\
P(A I)\end{array}$ & $\begin{array}{l}\text { Tugcu and Tiwari (2016), Liu } \\
\text { et al. (2019), Rahbar et al. } \\
\text { (2016) and Vyhmeister et al. } \\
\text { (2017) }\end{array}$ & $\begin{array}{l}\text { SDG } \\
12.5\end{array}$ & $\begin{array}{l}\text { P \& } \\
P(A I)\end{array}$ & $\begin{array}{l}\text { Nizami et al. (2017) and } \\
\text { Al-Hamamre et al. (2017) }\end{array}$ & $\begin{array}{l}\text { SDG } \\
16.9\end{array}$ & $\mathrm{~N} / \mathrm{A}$ & - \\
\hline $\begin{array}{l}\text { SDG } \\
3.5\end{array}$ & $\mathrm{~N} / \mathrm{A}$ & - & $\begin{array}{l}\text { SDG } \\
8.3\end{array}$ & $\mathrm{P}$ & $\begin{array}{l}\text { Ferroukhi et al. (2016b), } \\
\text { Bulavskaya and Reynès } \\
\text { (2018) and Ram et al. (2020) }\end{array}$ & $\begin{array}{l}\text { SDG } \\
12.6\end{array}$ & $\mathrm{~N} / \mathrm{A}$ & - & $\begin{array}{l}\text { SDG } \\
16.10\end{array}$ & N/A & - \\
\hline $\begin{array}{l}\text { SDG } \\
3.6\end{array}$ & $\mathrm{~N} / \mathrm{A}$ & - & $\begin{array}{l}\text { SDG } \\
8.4\end{array}$ & $\mathrm{~N} / \mathrm{A}$ & - & $\begin{array}{l}\text { SDG } \\
12.7\end{array}$ & $\mathrm{~N} / \mathrm{A}$ & - & $\begin{array}{l}\text { SDG } \\
16.11\end{array}$ & $\mathrm{~N} / \mathrm{A}$ & - \\
\hline $\begin{array}{l}\text { SDG } \\
3.7\end{array}$ & $\mathrm{~N} / \mathrm{A}$ & - & $\begin{array}{l}\text { SDG } \\
8.5\end{array}$ & $\mathrm{~N} / \mathrm{A}$ & - & $\begin{array}{l}\text { SDG } \\
12.8\end{array}$ & $\mathrm{~N} / \mathrm{A}$ & - & $\begin{array}{l}\text { SDG } \\
16.12\end{array}$ & $\mathrm{~N} / \mathrm{A}$ & - \\
\hline $\begin{array}{l}\text { SDG } \\
3.8 \\
\end{array}$ & $\mathrm{~N} / \mathrm{A}$ & - & $\begin{array}{l}\text { SDG } \\
8.6\end{array}$ & $\mathrm{P}$ & As 4.6 & $\begin{array}{l}\text { SDG } \\
12.9 \\
\end{array}$ & $P$ & $\begin{array}{l}\text { Owusu and } \\
\text { Asumadu-Sarkodie (2016) }\end{array}$ & & & \\
\hline $\begin{array}{l}\text { SDG } \\
3.9\end{array}$ & $\begin{array}{l}\mathrm{P}, \mathrm{N}, \\
P(A I)\end{array}$ & $\begin{array}{l}\text { Olatomiwa et al. (2016), } \\
\text { Nazir et al. (2019), Freiberg } \\
\text { et al. (2018), Nie et al. } \\
\text { (2018) and Wagh and } \\
\text { Kulkarni (2018) }\end{array}$ & $\begin{array}{l}\text { SDG } \\
8.7\end{array}$ & $\mathrm{~N} / \mathrm{A}$ & - & $\begin{array}{l}\text { SDG } \\
12.10\end{array}$ & $P$ & $\begin{array}{l}\text { Mandryk et al. (2016) and } \\
\text { Dalton et al. (2008) }\end{array}$ & $\begin{array}{l}\text { SDG } \\
17.1\end{array}$ & $\begin{array}{l}\mathrm{P}, \mathrm{N}, \\
P(A I)\end{array}$ & $\begin{array}{l}\text { Martinot et al. (2002), Surana } \\
\text { and Anadon (2015) and } \\
\text { Ragosa and Warren (2019) }\end{array}$ \\
\hline $\begin{array}{l}\text { SDG } \\
3.10\end{array}$ & $\mathrm{~N} / \mathrm{A}$ & - & $\begin{array}{l}\text { SDG } \\
8.8\end{array}$ & $\mathrm{~N} / \mathrm{A}$ & - & $\begin{array}{l}\text { SDG } \\
12.11\end{array}$ & $\begin{array}{l}\text { P \& } \\
P(A I)\end{array}$ & $\begin{array}{l}\text { Jha et al. (2017) and Rahbar } \\
\text { et al. (2016) }\end{array}$ & $\begin{array}{l}\text { SDG } \\
17.2\end{array}$ & $\mathrm{~N} / \mathrm{A}$ & \\
\hline
\end{tabular}

(continued on next page)

the economy, environment, and society (Giddings et al., 2002; Savelyeva and Douglas, 2017). Classifying the groups into the three domains enables the authors to have an accurate overview of the broad areas that impact RE utilization and AI-based RE implementation. We also presented the outcomes obtained (Fig. 3) by weighting the appropriateness of the evidence provided in 
Table 1 (continued).

\begin{tabular}{|c|c|c|c|c|c|c|c|c|c|c|c|}
\hline $\begin{array}{l}\text { Tar- } \\
\text { get }\end{array}$ & Impact & Ref. & $\begin{array}{l}\text { Tar- } \\
\text { get }\end{array}$ & Impact & Ref. & Target & Impact & Ref. & Target & Impact & Ref. \\
\hline $\begin{array}{l}\text { SDG } \\
3.11\end{array}$ & $\mathrm{~N} / \mathrm{A}$ & - & $\begin{array}{l}\text { SDG } \\
8.9\end{array}$ & $P$ & $\begin{array}{l}\text { Mandryk et al. (2016) and } \\
\text { Petrevska et al. (2016) }\end{array}$ & $\begin{array}{l}\text { SDG } \\
13.1\end{array}$ & $\begin{array}{l}\mathrm{P}, \mathrm{N}, \\
P(A I)\end{array}$ & $\begin{array}{l}\text { Abdolrasol et al. (2018), } \\
\text { Solaun and Cerdá (2019), } \\
\text { Alvarez-Herranz et al. (2017), } \\
\text { Rahman et al. (2017) and } \\
\text { Pino et al. (2019) }\end{array}$ & $\begin{array}{l}\text { SDG } \\
17.3\end{array}$ & $P$ & $\begin{array}{l}\text { Surana and Anadon (2015) } \\
\text { and Gabriel and Kirkwood } \\
\text { (2016) }\end{array}$ \\
\hline $\begin{array}{l}\text { SDG } \\
3.12\end{array}$ & $\mathrm{~N} / \mathrm{A}$ & - & $\begin{array}{l}\text { SDG } \\
8.10\end{array}$ & $\mathrm{~N} / \mathrm{A}$ & - & $\begin{array}{l}\text { SDG } \\
13.2\end{array}$ & $\begin{array}{l}\mathrm{P} \& \\
P(A I)\end{array}$ & $\begin{array}{l}\text { Hansen et al. (2019), } \\
\text { Re-thinking } 2050(2015) \text { and } \\
\text { Esteban et al. (2018) }\end{array}$ & $\begin{array}{l}\text { SDG } \\
17.4\end{array}$ & $\mathrm{~N} / \mathrm{A}$ & - \\
\hline $\begin{array}{l}\text { SDG } \\
3.13\end{array}$ & $\mathrm{~N} / \mathrm{A}$ & - & $\begin{array}{l}\text { SDG } \\
8.11\end{array}$ & $\mathrm{~N} / \mathrm{A}$ & - & $\begin{array}{l}\text { SDG } \\
13.3\end{array}$ & $\mathrm{~N} / \mathrm{A}$ & - & $\begin{array}{l}\text { SDG } \\
17.5\end{array}$ & $\begin{array}{l}\mathrm{P} \& \\
P(A I)\end{array}$ & $\begin{array}{l}\text { Ragosa and Warren (2019), } \\
\text { Kaygusuz (2012) and } \\
\text { Tabanjat et al. (2018) }\end{array}$ \\
\hline $\begin{array}{l}\text { SDG } \\
4.1\end{array}$ & $\begin{array}{l}\mathrm{P} \& \\
P(A I)\end{array}$ & $\begin{array}{l}\text { Giday (2014), Mauser et al. } \\
\text { (2016), Jin et al. (2017) and } \\
\text { Chel and Kaushik (2018) }\end{array}$ & $\begin{array}{l}\text { SDG } \\
8.12\end{array}$ & $\begin{array}{l}\text { P \& } \\
P(A I)\end{array}$ & $\begin{array}{l}\text { Aceleanu et al. (2015), } \\
\text { Halaweh (2018) and Shirley } \\
\text { et al. (2019) }\end{array}$ & $\begin{array}{l}\text { SDG } \\
13.4\end{array}$ & $\mathrm{P}$ & $\begin{array}{l}\text { Callithen and Matthew } \\
\text { (2007) and Padilla and } \\
\text { Hudson (2019) }\end{array}$ & $\begin{array}{l}\text { SDG } \\
17.6\end{array}$ & $\begin{array}{l}\mathrm{P} \& \\
P(A I)\end{array}$ & $\begin{array}{l}\text { Al-Shetwi et al. (2020b), } \\
\text { Re-thinking } 2050(2015) \text { and } \\
\text { Robles et al. (2019) }\end{array}$ \\
\hline $\begin{array}{l}\text { SDG } \\
4.2\end{array}$ & $\begin{array}{l}\mathrm{P} \& \\
P(A I)\end{array}$ & As 4.1 & $\begin{array}{l}\text { SDG } \\
9.1\end{array}$ & $P$ & $\begin{array}{l}\text { Bagheri et al. (2018) and } \\
\text { Kwasinski and Kwasinski } \\
\text { (2015) }\end{array}$ & $\begin{array}{l}\text { SDG } \\
13.5\end{array}$ & $\begin{array}{l}\mathrm{P} \& \\
P(A I)\end{array}$ & $\begin{array}{l}\text { Owusu and } \\
\text { Asumadu-Sarkodie (2016), } \\
\text { Kim et al. (2017) and } \\
\text { Harkouss et al. (2019) }\end{array}$ & $\begin{array}{l}\text { SDG } \\
17.7\end{array}$ & $\mathrm{P}$ & $\begin{array}{l}\text { Callithen and Matthew } \\
\text { (2007), Dóci and Gotchev } \\
\text { (2016) and Atabi (2004) }\end{array}$ \\
\hline $\begin{array}{l}\text { SDG } \\
4.3\end{array}$ & $\begin{array}{l}\text { P \& } \\
P(A I)\end{array}$ & As 4.1 & $\begin{array}{l}\text { SDG } \\
9.2\end{array}$ & $P$ & As 8.3 and 7.5 & $\begin{array}{l}\text { SDG } \\
14.1\end{array}$ & P \& N & $\begin{array}{l}\text { Kotrikla et al. (2017) and } \\
\text { Diab et al. (2016) }\end{array}$ & $\begin{array}{l}\text { SDG } \\
17.8\end{array}$ & $\mathrm{~N} / \mathrm{A}$ & - \\
\hline $\begin{array}{l}\text { SDG } \\
4.4\end{array}$ & $\begin{array}{l}\mathrm{P}, \mathrm{N}, \\
P(A I)\end{array}$ & $\begin{array}{l}\text { Global Status Report (2021), } \\
\text { Del Rio and Burguillo (2009), } \\
\text { Wei et al. (2010), Carley and } \\
\text { Konisky (2020), Colombo } \\
\text { et al. (2018) and Lucas et al. } \\
\text { (2018) }\end{array}$ & $\begin{array}{l}\text { SDG } \\
9.3\end{array}$ & $P$ & $\begin{array}{l}\text { Martinot et al. (2002) and } \\
\text { Pfeiffer and Mulder (2013) }\end{array}$ & $\begin{array}{l}\text { SDG } \\
14.2\end{array}$ & $P \& N$ & $\begin{array}{l}\text { Gasparatos et al. (2017), } \\
\text { Diab et al. (2016) and } \\
\text { Willsteed et al. (2017) }\end{array}$ & $\begin{array}{l}\text { SDG } \\
17.9\end{array}$ & $\mathrm{~N} / \mathrm{A}$ & - \\
\hline $\begin{array}{l}\text { SDG } \\
4.5\end{array}$ & $\mathrm{~N} / \mathrm{A}$ & - & $\begin{array}{l}\text { SDG } \\
9.4\end{array}$ & $\begin{array}{l}\text { P \& } \\
P(A I)\end{array}$ & $\begin{array}{l}\text { Wang et al. (2015), Ma et al. } \\
\text { (2014) and Halabi and } \\
\text { Mekhilef (2018) }\end{array}$ & $\begin{array}{l}\text { SDG } \\
14.3\end{array}$ & $\begin{array}{l}\mathrm{P}, \mathrm{N}, \\
P(A I)\end{array}$ & $\begin{array}{l}\text { Hu et al. (2018), Wagh and } \\
\text { Kulkarni (2018) and } \\
\text { Clements and Chopin (2017) }\end{array}$ & $\begin{array}{l}\text { SDG } \\
17.10\end{array}$ & $\mathrm{~N} / \mathrm{A}$ & - \\
\hline $\begin{array}{l}\text { SDG } \\
4.6\end{array}$ & $\begin{array}{l}\mathrm{P} \& \\
P(A I)\end{array}$ & $\begin{array}{l}\text { Chandel et al. (2016), } \\
\text { Bagheri et al. (2018) and } \\
\text { Ram et al. (2020) }\end{array}$ & $\begin{array}{l}\text { SDG } \\
9.5\end{array}$ & $\begin{array}{l}\text { P \& } \\
P(A I)\end{array}$ & $\begin{array}{l}\text { Karmaker et al. (2018) and } \\
\text { Mohammed et al. (2017) }\end{array}$ & $\begin{array}{l}\text { SDG } \\
14.4\end{array}$ & $P \& N$ & $\begin{array}{l}\text { Langhamer (2012) and Hagos } \\
(2007)\end{array}$ & $\begin{array}{l}\text { SDG } \\
17.11\end{array}$ & $\mathrm{~N} / \mathrm{A}$ & - \\
\hline $\begin{array}{l}\text { SDG } \\
4.7\end{array}$ & $\mathrm{~N} / \mathrm{A}$ & - & $\begin{array}{l}\text { SDG } \\
9.6\end{array}$ & $\begin{array}{l}\mathrm{P}, \mathrm{N}, \\
\mathrm{P}(A I)\end{array}$ & $\begin{array}{l}\text { Azimoh et al. (2017) and } \\
\text { Fashina et al. (2019) }\end{array}$ & $\begin{array}{l}\text { SDG } \\
14.5\end{array}$ & $\mathrm{~N} / \mathrm{A}$ & - & $\begin{array}{l}\text { SDG } \\
17.12\end{array}$ & $\mathrm{P}$ & $\begin{array}{l}\text { Griffith-Jones et al. (2017), } \\
\text { Hymel (2006) and Simsek } \\
\text { and Simsek (2013) }\end{array}$ \\
\hline $\begin{array}{l}\text { SDG } \\
4.8\end{array}$ & $\mathrm{~N} / \mathrm{A}$ & - & $\begin{array}{l}\text { SDG } \\
9.7\end{array}$ & $\mathrm{~N} / \mathrm{A}$ & - & $\begin{array}{l}\text { SDG } \\
14.6\end{array}$ & $\mathrm{~N} / \mathrm{A}$ & - & $\begin{array}{l}\text { SDG } \\
17.13 \\
\end{array}$ & $\mathrm{~N} / \mathrm{A}$ & - \\
\hline $\begin{array}{l}\text { SDG } \\
4.9\end{array}$ & $\mathrm{~N}$ & $\begin{array}{l}\text { Lucas et al. (2018) and } \\
\text { United Nations Educational } \\
\text { and Organization (2020) }\end{array}$ & $\begin{array}{l}\text { SDG } \\
9.8\end{array}$ & $\mathrm{~N} / \mathrm{A}$ & - & $\begin{array}{l}\text { SDG } \\
14.7\end{array}$ & $\mathrm{~N} / \mathrm{A}$ & - & $\begin{array}{l}\text { SDG } \\
17.14\end{array}$ & $\begin{array}{l}\mathrm{P} \& \\
P(A I)\end{array}$ & $\begin{array}{l}\text { Al-Shetwi et al. (2020b), } \\
\text { Re-thinking 2050 (2015), } \\
\text { Fraundorfer and Rabitz } \\
\text { (2020) and Collste et al. } \\
(2017)\end{array}$ \\
\hline $\begin{array}{l}\text { SDG } \\
4.10\end{array}$ & $\mathrm{P}$ & Adenle (2020) & $\begin{array}{l}\text { SDG } \\
10.1\end{array}$ & $\mathrm{~N} / \mathrm{A}$ & - & $\begin{array}{l}\text { SDG } \\
14.8\end{array}$ & $\mathrm{P}$ & $\begin{array}{l}\text { Wright (2019) and Zvezdov } \\
\text { (2020) }\end{array}$ & $\begin{array}{l}\text { SDG } \\
17.15\end{array}$ & $\mathrm{~N} / \mathrm{A}$ & - \\
\hline $\begin{array}{l}\text { SDG } \\
5.1\end{array}$ & $\mathrm{~N} / \mathrm{A}$ & - & $\begin{array}{l}\text { SDG } \\
10.2\end{array}$ & $P$ & $\begin{array}{l}\text { Nelson and Kuriakose (2017) } \\
\text { and Madriz-Vargas et al. } \\
(2018)\end{array}$ & $\begin{array}{l}\text { SDG } \\
14.9\end{array}$ & $\mathrm{~N} / \mathrm{A}$ & - & $\begin{array}{l}\text { SDG } \\
17.16\end{array}$ & $P \& N$ & $\begin{array}{l}\text { Atabi (2004), Bugaje (2006) } \\
\text { and Eitan et al. (2019) }\end{array}$ \\
\hline $\begin{array}{l}\text { SDG } \\
5.2\end{array}$ & $\mathrm{~N} / \mathrm{A}$ & - & $\begin{array}{l}\text { SDG } \\
10.3 \\
\end{array}$ & $\mathrm{~N} / \mathrm{A}$ & - & $\begin{array}{l}\text { SDG } \\
14.10 \\
\end{array}$ & $\mathrm{~N} / \mathrm{A}$ & - & $\begin{array}{l}\text { SDG } \\
17.17\end{array}$ & $P$ & $\begin{array}{l}\text { Sovacool (2013), Atabi (2004) } \\
\text { and Zamfir (2012) }\end{array}$ \\
\hline $\begin{array}{l}\text { SDG } \\
5.3\end{array}$ & $\mathrm{~N} / \mathrm{A}$ & - & $\begin{array}{l}\text { SDG } \\
10.4\end{array}$ & $P$ & Abdmouleh et al. (2015) & $\begin{array}{l}\text { SDG } \\
15.1\end{array}$ & $\mathrm{~N}$ & $\begin{array}{l}\text { Gasparatos et al. (2017), } \\
\text { Schuster et al. (2015) and } \\
\text { Frid et al. (2012) }\end{array}$ & $\begin{array}{l}\text { SDG } \\
17.18\end{array}$ & $\mathrm{~N} / \mathrm{A}$ & - \\
\hline $\begin{array}{l}\text { SDG } \\
5.4\end{array}$ & $\mathrm{P}$ & $\begin{array}{l}\text { Kirsanova et al. (2018) and } \\
\text { Amador (2017) }\end{array}$ & $\begin{array}{l}\text { SDG } \\
10.5\end{array}$ & $P$ & Onifade (2016) & $\begin{array}{l}\text { SDG } \\
15.2\end{array}$ & $\begin{array}{l}\mathrm{P}, \mathrm{N}, \\
P(A I)\end{array}$ & $\begin{array}{l}\text { Poggi et al. (2018), Schuster } \\
\text { et al. (2015) and Turney and } \\
\text { Fthenakis (2011) }\end{array}$ & $\begin{array}{l}\text { SDG } \\
17.19\end{array}$ & $\mathrm{~N} / \mathrm{A}$ & - \\
\hline $\begin{array}{l}\text { SDG } \\
5.5\end{array}$ & $\mathrm{~N} / \mathrm{A}$ & - & $\begin{array}{l}\text { SDG } \\
10.6\end{array}$ & $\mathrm{~N} / \mathrm{A}$ & - & $\begin{array}{l}\text { SDG } \\
15.3\end{array}$ & $\mathrm{~N} / \mathrm{A}$ & - & & & \\
\hline
\end{tabular}

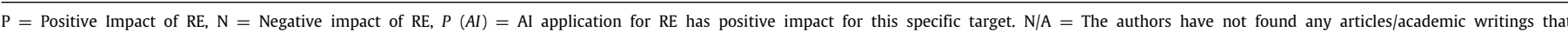
relate $\mathrm{RE}$ and Al-based with this target goal up to date.

Table 2

Results of SDG's target analysis with percentage (\%).

\begin{tabular}{|c|c|c|c|c|c|c|c|c|c|c|}
\hline No. & The goal & $\begin{array}{l}\text { No of } \\
\text { targets }\end{array}$ & $\begin{array}{l}\text { Positive } \\
\text { impact }\end{array}$ & $\%$ & $\begin{array}{l}\text { Negative } \\
\text { impact }\end{array}$ & $\%$ & $\begin{array}{l}\text { Is there any } \\
\text { role for } \mathrm{AI} ?\end{array}$ & $\%$ & No evidence & $\%$ \\
\hline 1 & No poverty & 7 & 5 & 71.42 & 2 & 28.5 & 0 & 0 & 2 & 28.57 \\
\hline 2 & Zero Hunger & 8 & 3 & 37.50 & 1 & 12.5 & 2 & 25 & 5 & 62.5 \\
\hline 3 & Good health and well-being & 13 & 2 & 15.38 & 1 & 7.69 & 1 & 7.69 & 11 & 84.61 \\
\hline 4 & Quality education & 10 & 6 & 60 & 2 & 20 & 5 & 50 & 3 & 30 \\
\hline 5 & Gender equality & 9 & 1 & 11.11 & 0 & 0 & 0 & 0 & 8 & 88.88 \\
\hline 6 & Clean water and sanitation & 8 & 4 & 50 & 1 & 12.5 & 4 & 50 & 3 & 37.5 \\
\hline 7 & Affordable and clean energy & 5 & 5 & 100 & 0 & 0 & 5 & 100 & 0 & 0 \\
\hline 8 & Decent work and economic growth & 12 & 6 & 50 & 1 & 8.33 & 2 & 16.66 & 6 & 50 \\
\hline 9 & Industry, Innovation and infrastructure & 8 & 6 & 75 & 1 & 12.5 & 3 & 37.5 & 2 & 25 \\
\hline 10 & Reducing inequality & 10 & 3 & 30 & 0 & 0 & 0 & 0 & 7 & 70 \\
\hline 11 & Sustainable cities and communities & 10 & 6 & 60 & 3 & 30 & 6 & 60 & 4 & 40 \\
\hline 12 & Responsible consumption and production & 11 & 6 & 54.54 & 1 & 9.09 & 4 & 36.36 & 5 & 45.45 \\
\hline 13 & Climate action & 5 & 4 & 80 & 1 & 20 & 3 & 60 & 1 & 20 \\
\hline 14 & Life below water & 10 & 5 & 50 & 4 & 40 & 1 & 10 & 5 & 50 \\
\hline 15 & Life on land & 12 & 3 & 25 & 5 & 41.6 & 2 & 16.66 & 7 & 58.33 \\
\hline 16 & Peace, Justice, and Strong institutions & 12 & 1 & 8.33 & 1 & 8.33 & 0 & 0 & 11 & 91.66 \\
\hline 17 & Partnerships for the goals & 19 & 9 & 47.36 & 3 & 15.7 & 4 & 21.05 & 10 & 52.63 \\
\hline \multicolumn{2}{|c|}{ Total } & 169 & 75 & $44.3 \%$ & 27 & $15.97 \%$ & 42 & $24.85 \%$ & 90 & $53.2 \%$ \\
\hline
\end{tabular}




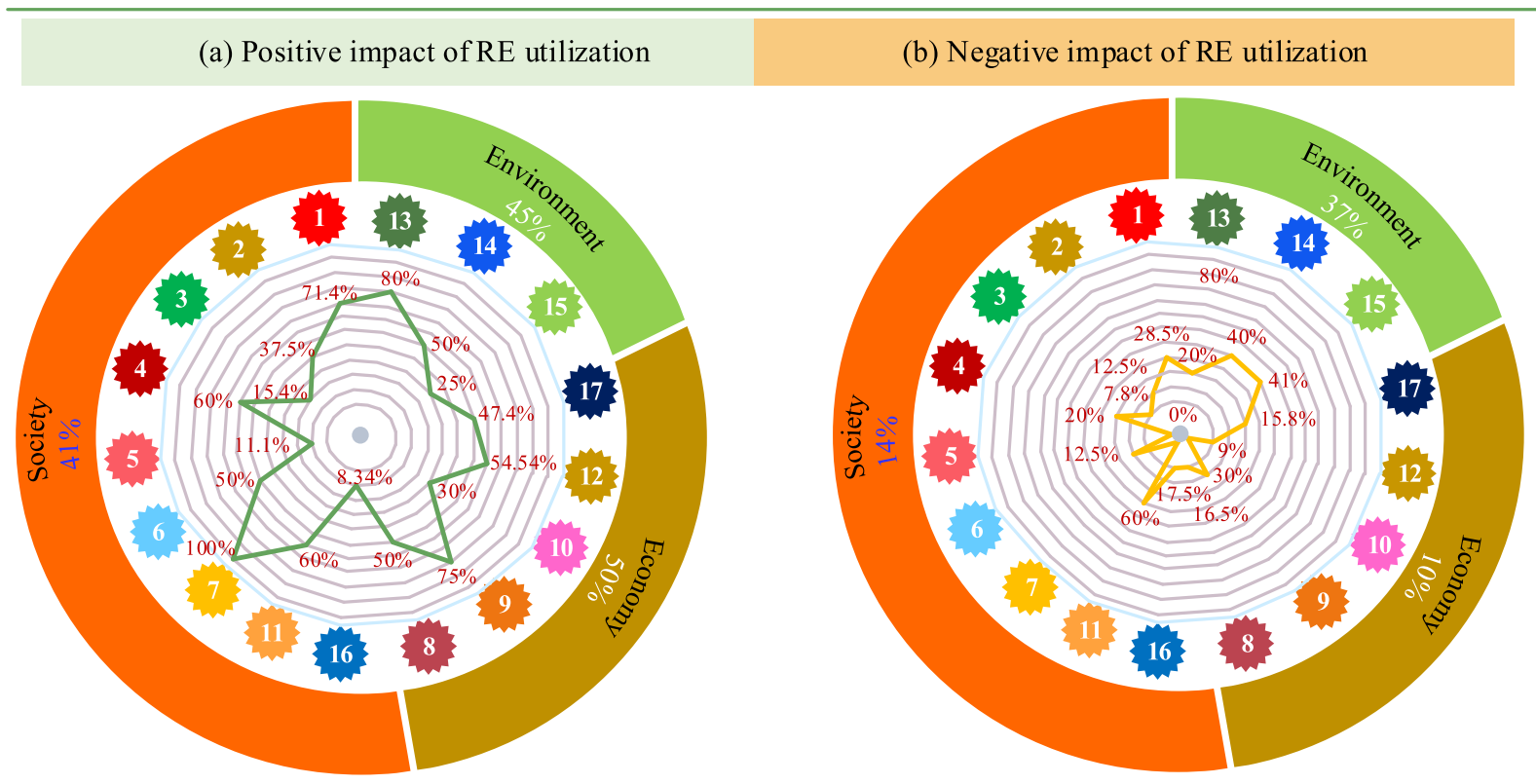

(c) Role of AI as enabler for RE utilization towards achieving SDGs

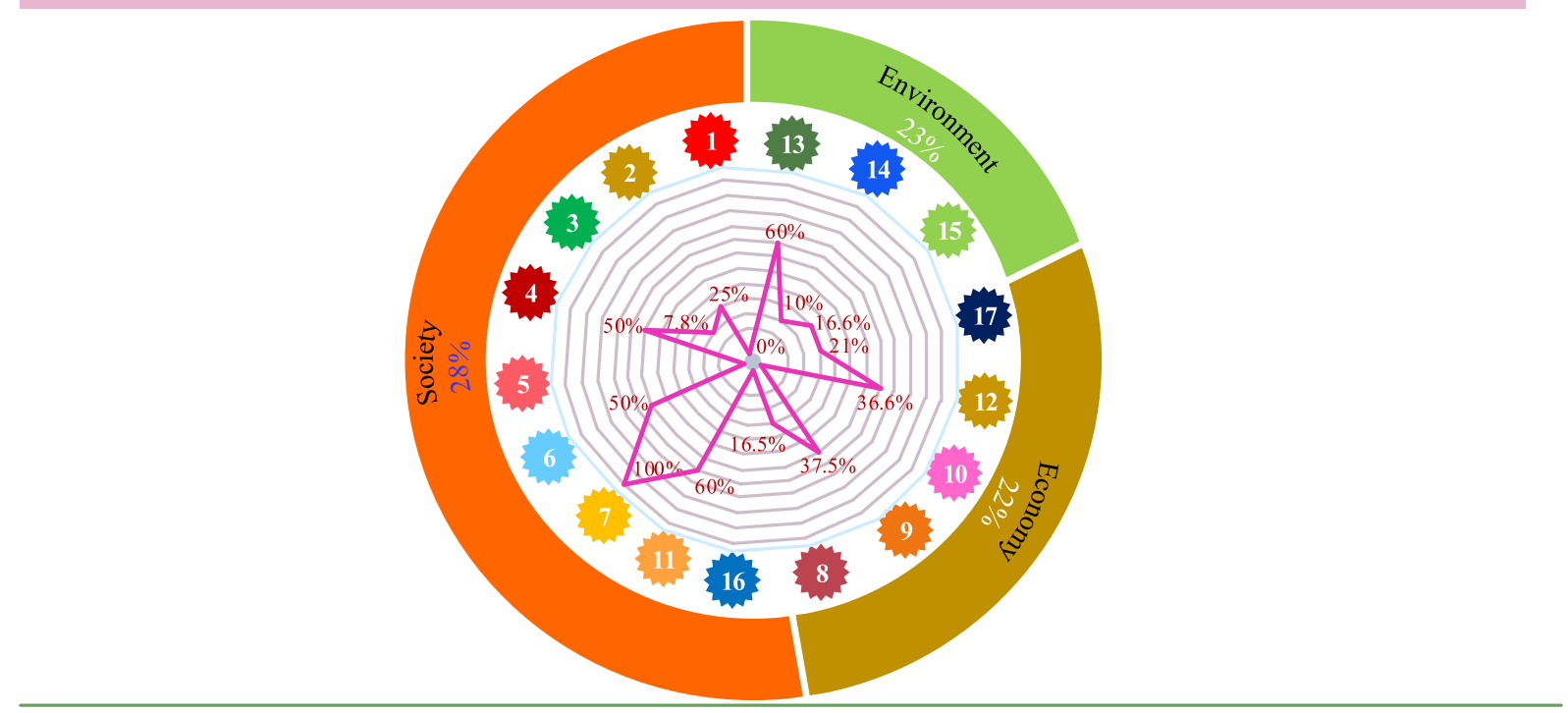

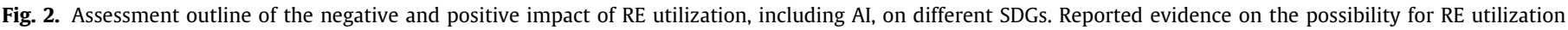
on the 17 SDGs as (a) positive impact, (b) negative impact, and (c) application of AI for RE as an enabler.

every reference to evaluate an affinity (the interlinking) with respect to the targets assessed (in percentage), as explained in Section 2.

\subsection{RE utilization and environmental outcomes}

Most researchers agreed that the RE projects had enhanced environmental impacts. For example, carbon dioxide gas elimination and climate change understanding within the world population and contribute toward achieving the related SDGs effectively (Buonocore et al., 2019; Muh et al., 2018). However, RE utilization with environmental impact has limited negative effects, especially in marine renewable sources (Shields et al., 2011; Miao, 2014). In this context, our assessment of relevant evidence indicates that within the environment group, 45 targets (45\%) must benefit from the development and utilization of RE sources (Fig. 4). For instance, in SDG 13, on climate change, RE can act as a positive impact toward achieving $80 \%$ of its target effectively. Based on the evidence, the RE sources could act as an enabler toward achieving 50\% and 25\% of the SDG 13 and SDG 14 targets, respectively. Notably, the positive impact ratio (45\%) would increase because of the continuous development of RE sources and its application to cover many sectors and address certain present limitations. Benefits from RE for environment group (SDG13, SDG14, and SDG15) can be summarized on its ability to reduce the GHG produced from traditional fuels (Solaun and Cerdá, 2019); minimize some kinds of air pollution (AlvarezHerranz et al., 2017); reduce climate-related hazards, such as $\mathrm{CO}_{2}$ emissions (Khayyam and Nazar, 2021); reduce the effects of ocean acidification (Clements and Chopin, 2017); and many other benefits to the life, land, water, and forests to maintain the ecosystem (Oakleaf et al., 2019; Konietzko et al., 2020). For instance, looking at targets 13.1 and 13.2 , which call to climaterelated hazards and incorporate action on climate change into national strategies, strategies, and planning. Many evidence confirmed that the utilization of RE resources would reduce the $\mathrm{CO}_{2}$ emission in the power sector to approximately $70 \%$ by 2050 , according to the plan published in the US (Khanna et al., 2016), 


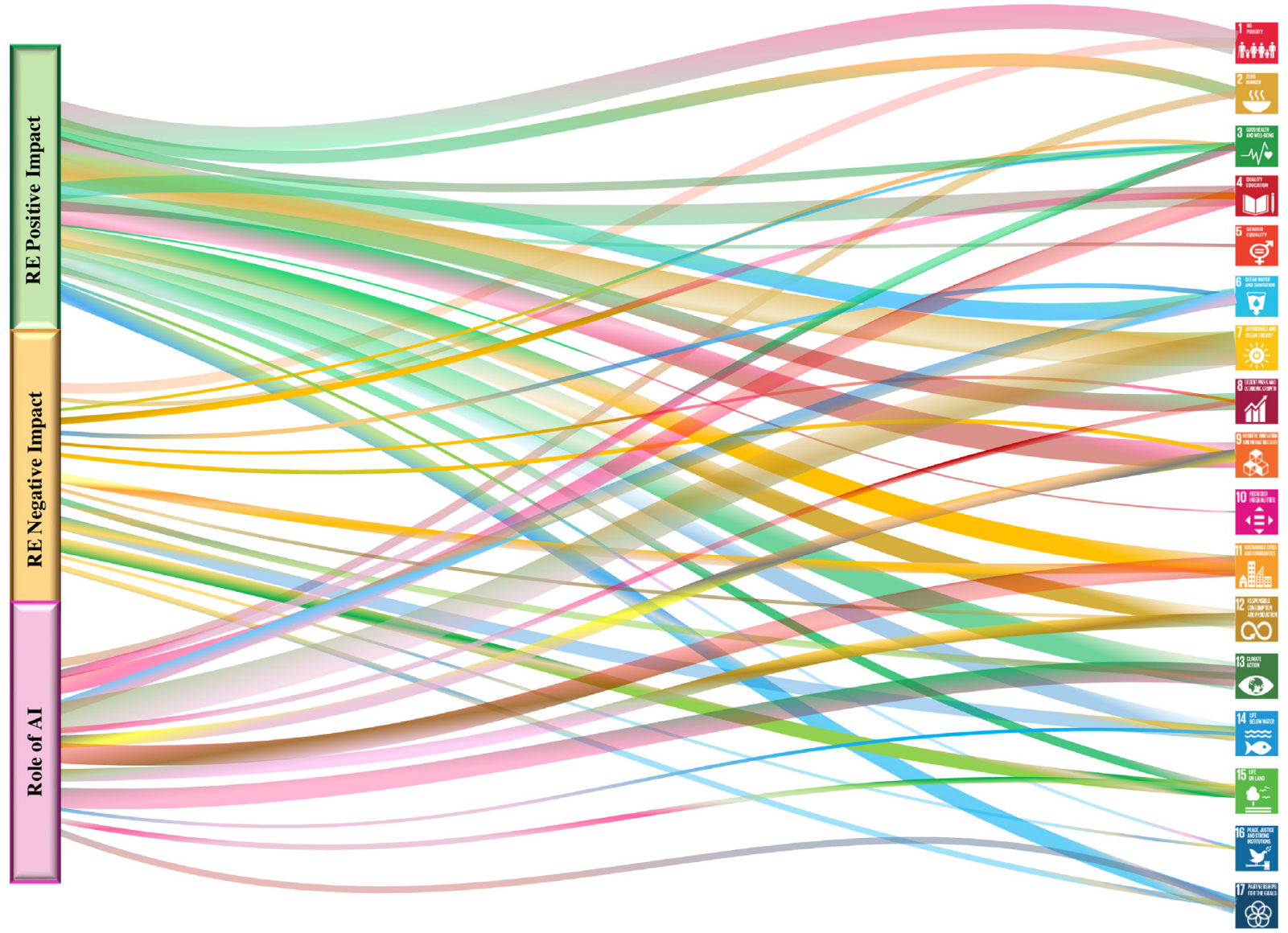

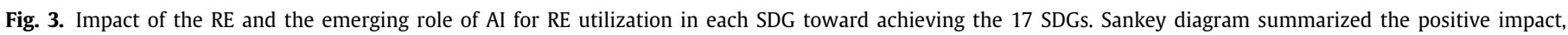
negative impact, and the role of AI for RE utilization for all SDGs. Thick and thin lines correspond to the high and low impact of each SDGs.

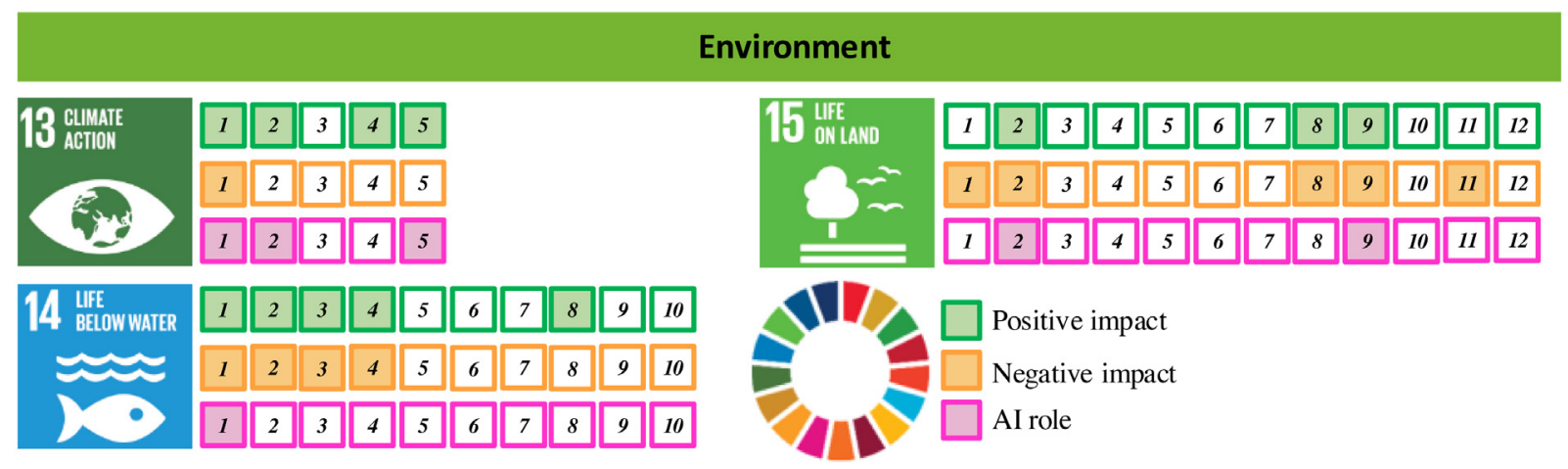

Fig. 4. Thorough evaluation of the effect of RE utilization at the SDGs within the environment group (SDGs 13, 14, and 15).

New Zealand (Walmsley et al., 2014), and Europe (Walmsley et al., 2014). Additionally, to enhance the condition of ecosystems, AI applications can play an important role in helping RE in this regard. For instance, using some optimization methods will reduce the emission (target 15.1) in the MG system that consists of five different RE sources to $8.1 \%$ using backtracking search algorithm (Abdolrasol et al., 2018) and to $8.4 \%$ using particle swarm optimization (Hannan et al., 2019) in comparison to that without using these methods. One more example is target 15.2, which aims to manage all types of forests. This target can benefit from using AI mapping techniques for RE potential maps to predict the proper locations for the expansion of RE source-based facilities and land protection. The optimized machine learning technique is used to enhance the battery-based RE systems to reduce its degradation and environmental impact (Hannan et al., 2020d, 2018) (13.1). Moreover, according to Dhunny et al. (2019), the genetic algorithm showed a good performance to find appropriate areas for the construction of wind turbines with negligible effects on forest, fauna, and flora, including migratory bird pathways, which is also consistent with target 15.9.

On the other hand, 10 targets of the SDGs within the environment group, which represent $37 \%$, may be negatively affected by RE development. However, their consideration is crucial. In this regard, the most negative impacts come from marine RE. In particular, tidal energy may act as an inhibitor for (15.4) target when established on coastal estuaries or bays, and tidal barrages can cause significant ecological effects on bird feeding areas (Frid et al., 2012). Moreover, wave energy and offshore 
tidal stream energy collectors, including mid-water column, floating, and seabed mounted devices, with a range of moving-part configurations, resulting in a particular complex of possible environmental impacts for each device category (Alipour et al., 2020b; Lin and Yu, 2012). Looking to target 14.2, a potential positive impact on this target would be the use of RE to produce clean energy and to protect, conserve marine's systems sustainably marine environment. Marine RE plays a fundamental role in reducing anthropogenic $\mathrm{CO}_{2}$ emissions. These attributes will provide a reference to protect sea environmental change and achieve productive oceans (Willsteed et al., 2017; Bonar et al., 2015). However, marine RE may have a negative effect toward achieving the target 14.2 because these sources may increase underwater noise and collision risk. During day-to-day operation, underwater noise, electromagnetic field emissions and collisions or avoidance with the energy constructions represent further possible influences on coastal species, particularly in large predators (Willsteed et al., 2017). Furthermore, the large-scale wind farm and solar energy in some countries up a large space, thereby leading to the cutting of trees and use of agricultural lands (Schuster et al., 2015; Turney and Fthenakis, 2011). In the same context, they act as an inhibitor of achieving target 15.2 and sub-target of 15.1 and 15.11 . Overall, the RE may harm forests, wetlands, mountains, and drylands. The existing evidence base is greater for some pathways (e.g., hydropower and bioenergy) than others (e.g., wind, solar, geothermal, and ocean), and the point remains that the large-scale distribution of RE may have some biodiversity tradeoffs (Gasparatos et al., 2017).

\subsection{RE utilization and social outcomes}

The development of RE may also positively affect the attainment of several SDGs in the Society group. In this context, from 82 targets within this group, 33 targets (41\%) can be benefited from the RE source development. However, negative impacts are identified with regard to 11 targets (41\%). Moreover, the emerging role of $\mathrm{AI}$ for RE utilization can contribute toward achieving 23 targets (28\%) (Fig. 5). Importantly, based on the classification of the 17 SDGs into three groups, SDGs within the Society group include SDGs (1-7), SDG 11, and SDG 16. Looking at SDG 7 (Affordable and Clean Energy), clear evidence shows that the utilization of RE sources will act as an enabler toward the achievement of all targets (100\%) within this goal without any negative impact. Moreover, the AI will affect the five targets (100\%) within SDG 7 positively because of its ability to improve the operation, efficiency, and development of RE to reduce energy costs and emissions. For instance, Ivanovski et al. reported that by 2050 , the costs of RE were projected to be much lower than non-RE (Ivanovski et al., 2021). Furthermore, the utilization of RE resources will reduce the $\mathrm{CO}_{2}$ emission in the power sector to approximately $70 \%$ by 2050 (Khanna et al., 2016). Concerning AI role, the digital and intelligent energy systems, drawing on the ever-increasing data on energy demand and supply, identifying who needs energy and who to supply it at the right time, in the right place, and at the lowest cost will be possible (Yang et al., 2019). Moreover, AI plays an important role in reducing cost according to smart scheduling based on the weather condition to guarantee the continuous power supply and enable consumers to respond to load management signals when operated under the supervision of a scheduling coordinator (Jones, 2018). For example, the fulfillment of target 7.2, calling to substantially boost the RE quota in the global energy mix, is strong evidence of the RE source utilization to achieve this target. In this regard, the share of RE in the global energy mix was $8.6 \%$ in 2010 and increased to $18.2 \%$ by the end of 2017 and then jumped to nearly $28 \%$ in the first quarter of 2020 from $26 \%$ in 2019 and expected to rise to $45 \%$ by 2040 . Furthermore, in 2018 , the RE produced at least 1 GW of generating capacity in more than 90 countries, whereas at least 30 countries exceeded $10 \mathrm{GW}$ of capacity across the globe (Global Status Report, 2021; International Energy Agency, 2020). These pieces of evidence confirmed without doubt that RE utilization contributes toward achieving this goal. In addition, toward achieving SDG 4, especially targets 4.1 and 4.4, this continuous growth of RE leads to the development of the industry and what RE's potential can mean for creating jobs and speeding up economic development (Global Status Report, 2021). Moreover, RE-based remote teaching (schools and institutes electrified by RE) would educate more students in underdeveloped countries with improved performance (Del Rio and Burguillo, 2009; Carley and Konisky, 2020). Several youth and adults can work in this sector and direct youth toward income-generating activities in this sector because of the establishment of RE factories and the prosperity of the RE industry (Wei et al., 2010). The RE development can have a positive and tangible impact on jobs because this energy is local in nature and can generally be made accessible without heavy infrastructure being available (Colombo et al., 2018). In sum, based on the published evidence, the following social benefits, namely, local employment, job opportunities, improved health, and consumer choice, can be achieved as result of RE development.

Moreover, AI could support the use of Smart Grids, which could increase the penetration of RE in the system (Ramchurn et al., 2012). One more example, the advancement of RE can contribute toward achieving target 6.4 via the use of solar PV energy and/or hybrid RE in pumping the water from the underground wells in the rural and desert areas and thus decrease the number of people who suffer from water shortages (Barron-Gafford et al., 2019; Bertsiou et al., 2018). The AI can also be used for managing the water pumping via an efficient way for irrigation systems using optimization methods as proved by Chaouali et al. (2018), who developed the fuzzy logic optimization to increase the efficient use of water and reduce water waste. One more example, the RE can act positively to target 6.1 calls to universal and equitable access to safe and affordable drinking water for all. In this regard, the World Health Organization (Bowen et al., 2020; Freire-Gormaly, 2018) estimates that 760 million people worldwide do not have access to clean drinking water. The areas with the greatest water scarcity are generally off-grid, remote, and have high solar irradiation. Therefore, a standalone hybrid $\mathrm{PV} /$ wind energy system is an effective solution to continuously power a submersible water pump (underground well) to produce drinking water (Freire-Gormaly, 2018). For SDG 11, the use of AI in smart cities is going to improve the cost-effectiveness of new and existing energy infrastructure and increase the quality of life (Yang et al., 0000). On the other hand, some evidence found that RE utilization may act as an inhibitor toward achieving some targets or sub-targets within the Society group, including 1.2, 1.4, $3.9,4.4,4.9,6.6,11.1,11.8$, and 16.2. However, no negative impact was found within SDG 2, SDG 5, and SDG 7 (Table 1). In this context, looking at target 3.9, called to decrease the diseases and deaths related to toxic substances and pollution of air, soil, and water, RE source, such as biomass may act as inhibitor for this target. This is because it releases carbon monoxide, leading to headaches, nausea, dizziness, and in high concentrations which may lead to premature death (Freiberg et al., 2018; Subramanian, 2014). One more example is related to target 6.6 , in which there is weak evidence that the installation of RE farms such as solar, wind, biomass, and hydropower may affect the water-related ecosystems such as forests, wetland, and mountain/valleys especially large scale RE farms (Gasparatos et al., 2017). For instance, cutting the wood for biomass energy operation, using the wetland for solar/wind farms installation, etc. (Gasparatos et al., 2017; Drechsler et al., 2017b). 


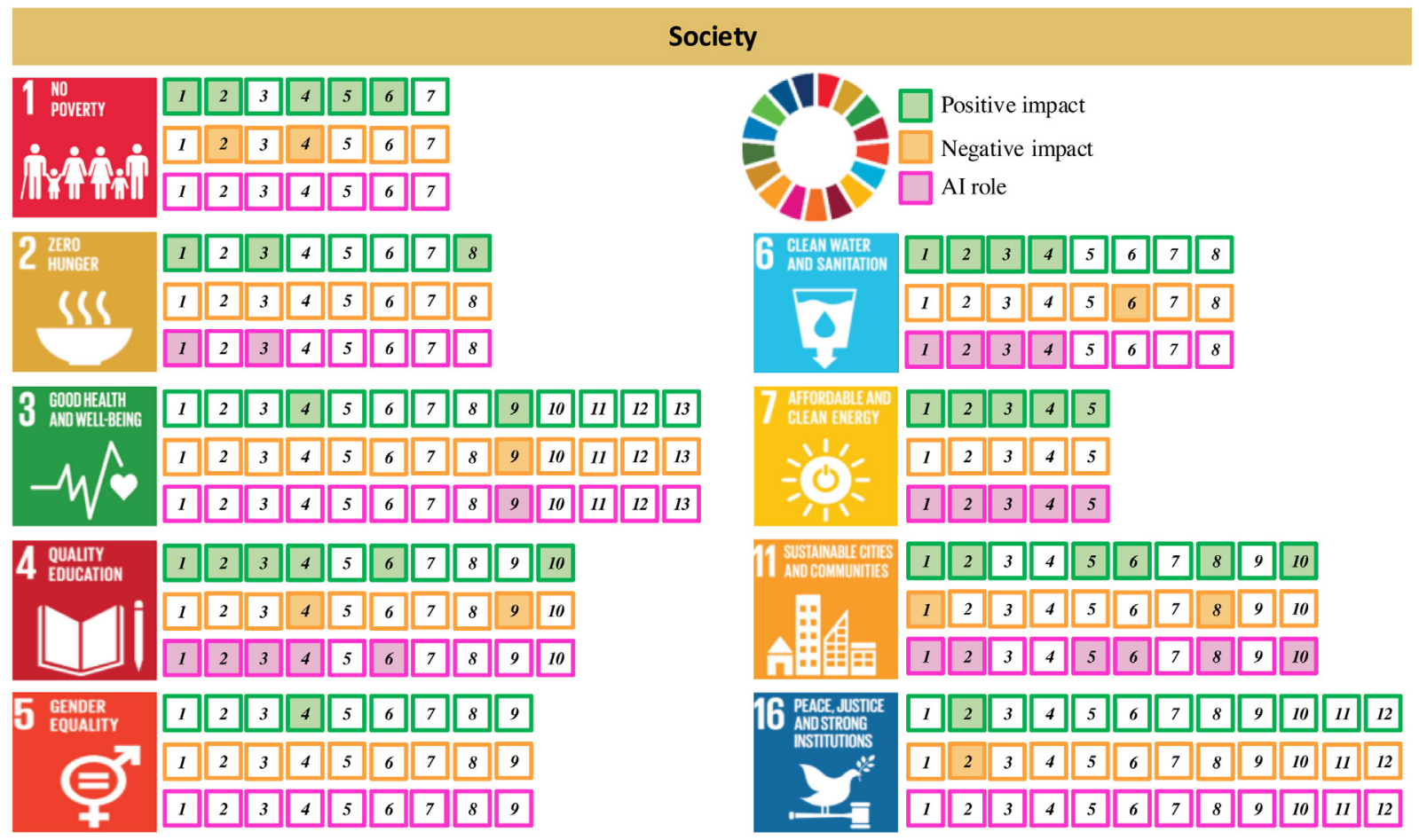

Fig. 5. Thorough evaluation of the effect of RE utilization at the SDGs within the Society group [SDGs (1-7), SDG 11 and SDG 16].

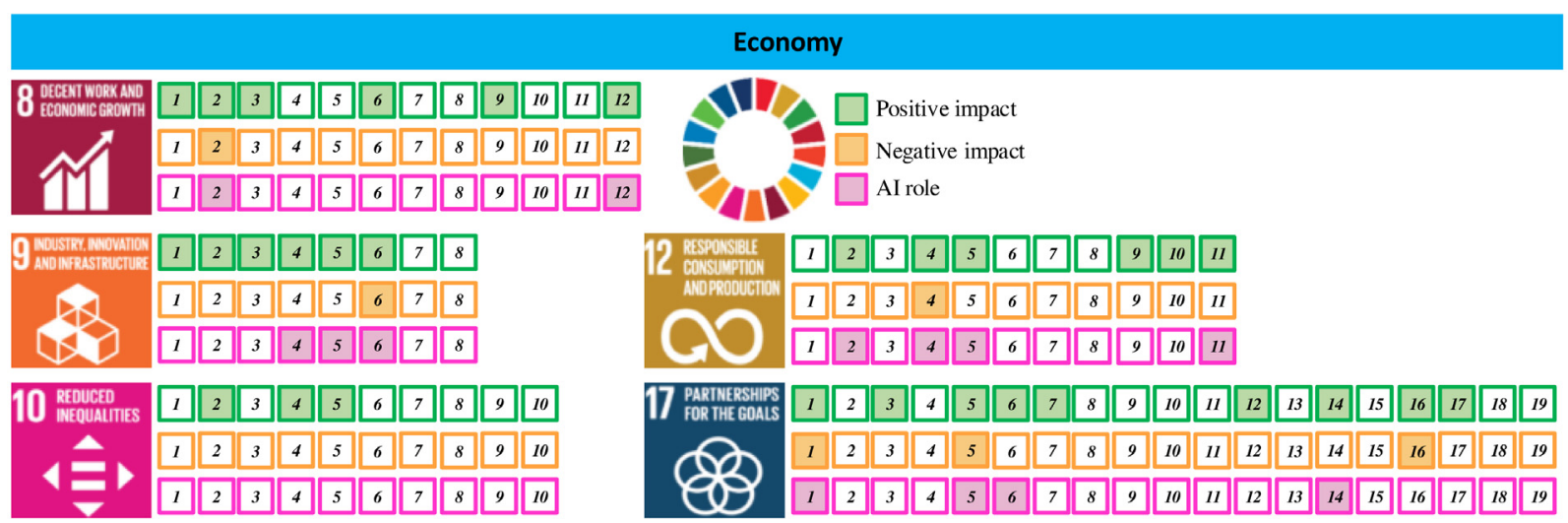

Fig. 6. Thorough evaluation on the effect of RE utilization at the SDGs within the Economy group [SDGs (8-10), SDG 12 and SDG 17].

\subsection{RE utilization and economic outcomes}

The remaining groups or categories of SDGs relevant to the Economy are described in Fig. 6. The five SDGs within the Economy group are relevant to decent work and economic growth, industry, innovation and infrastructure, reduced inequality, responsible consumption and production, and partnerships to achieve the goal (SDGs 8, 9, 10,12 and 17, respectively). Regarding the economic group, we have identified that the RE utilization could positively help achieve 30 targets (50\%) out of the 60 targets within this group.

The utilization and deployment of RE offer economic opportunities from using direct labor from remote communities, local materials and enterprises, local owners, and local banks' services. Furthermore, the projects of RE have facilitated the communities by setting up a trust fund to invest the money earned by the sale of energy to the local economy. Looking at SDG 8, economic growth, and decent work, the current studies demonstrate that increased RE deployment contributes to economic development and jobs in manufacturing and structures. Based on the policy intervention enacted in different nations, jobs may grow from a few thousand to more than one million by 2030 (Ferroukhi et al., 2016a). The jobs created due to RE development are expected to compensate for the loss of jobs in sectors, such as the fossil fuels sector because the ones involved in the supply chain of $\mathrm{RE}$ are typically more dispersed and labor intensive than the traditional energy market. For example, solar PV creates approximately twice the number of jobs compared with coal or natural gas for every unit of electricity produced (Essletzbichler, 2012). The achievement of target 8.3 can benefit from RE to create new jobs. For instance, the RE added approximately 7.7 million jobs across the globe in 2014, which China led with approximately 3390 jobs, based on IRENA report 2016 (IRENA, 2016). Likewise, the transition to RE may positively impact the Dutch Economy and is expected to create almost 50,000 new jobs by 2030 , adding almost 1\% of GDP (Bulavskaya and Reynès, 2018), which act positively on target 8.1. In line with these facts, the expected effects of the RE sources development on the economic sector for some countries, including European Union, USA, UK, Japan, Germany, 
Mexico, Netherland, Chile, and Saudi Arabia is investigated in detail in Ferroukhi et al. (2016a).

The RE options may considerably enhance the performance of rural small- and micro-enterprises. In this context, the use of RE emerges in the market for a rural house, biogas, and hydropower for rural entrepreneurship and government programs led to greater attention financing, involving small-scale market facilitation organizations, private power developer, and donor assistance, which may contribute toward achieving target 9.3 (Pfeiffer and Mulder, 2013). Many evidences found from our search confirm that the RE will positively impact innovation, industry, and infrastructure (SDG 9). Moreover, concerning SDG 17, the RE may help in achieving some targets within this goal, such as strengthening domestic resource mobilization as RE diversity of sources began to widespread application, including developing countries (Surana and Anadon, 2015), thereby offering a new policy of taxes with respect RE technology to encourage investment by citizens/individual or companies. Many organizations have established RE projects, such as PV power plants for rural and remote areas in developing countries (Freire-González and PuigVentosa, 2019), improving the global partnership for sustainable development as the RE sector witnessed global collaboration in terms of research, financial support, and economic aspect (Eitan et al., 2019).

The emerging role of AI for RE utilization may help it achieve some targets (22\%) within the economy group because $(a)$ the $\mathrm{AI}$, machine learning, and smart communication can be used to succeed this harmonization effort to have unique standards and requirements concerning $\mathrm{RE}$ integration around the world (AlShetwi et al., 2020b; Carley et al., 2018) (target 17.14 and 17.6); (b) improvement and optimization (using $\mathrm{AI}$ ) will enhance the RE efficiency and production (Nabavi-Pelesaraei et al., 2016; Martin and Saikawa, 2017) (target 12.11); (c) optimization of the west to energy (WtE) technologies for treating of various waste fractions in a medium-term future energy system considering their complex properties and optimizing both investments and production. Optimization of routes, which include waste components (i.e., food and yard wastes, non-biodegradable components, rubber, plastic, textile, leather, and wood) are the optimized WtE routes for maximum power generation potential by biochemical and thermochemical treatments of solid waste (Korai et al., 2016; Subiyanto et al., 2012) (targets 12.4, 12.5); and (d) the AI, machine learning, optimization, and smart communications have positive impact on increasing RE productivity, reduce the cost, and introduce innovation toward smart grid (Shen et al., 2020; Hannan et al., 2020c,a) (target 8.1). Furthermore, fewer targets within the Economy group ( 6 targets, $10 \%$ ) can be impacted negatively by RE. For instance, we have found evidence which confirmed that no remarkable causal link is found between RE consumption and total factor productivity (TFP) growth in the BRICS (Tugcu and Tiwari, 2016). Marine RE can have detrimental environmental effects because of the danger of collision, habitat destruction, noise, and electromagnetic fields of RE devices (Shields et al., 2011).

\section{Role of AI in RE utilization}

The emerging role of AI for RE utilization may help the latter to achieve some targets within the Environment, Society, and Economy groups by $22 \%, 28 \%$, and $23 \%$, respectively. This is due to the ability of AI to improve the operation and efficiency of RE sources and reduce the cost of operation and produced energy as well as minimize their environmental impacts efficiently (Jha et al., 2017; Srivastava, 2020; Hannan et al., 2020b). Given that the intermittency and ambiguity of RE supply are major concerns, emerging technologies, such as AI and machine learning provide plenty of opportunities to solve these concerns because they are primarily intended to process unknown data (Kalogirou, 2007). Moreover, in developed countries, the power sector has already begun to use AI and related technologies that enable smart grids, smart meters, and the Internet of Things (IoT) devices to interact. Such technology can help enhance energy management, efficiency, transparency, and the use of RE sources (Nižetić et al., 2020). This step helps the RE to achieve many targets (i.e., $100 \%$ of SDG 7 targets). Furthermore, the role of AI applications can cover different areas of RE system, such as forecasting, emission reduction, cost-minimizing, robust and smooth control, high power quality without fluctuation even when input is intermittent, expansion of novel technologies for the optimal production from available natural resources, awareness of the environment, enhanced energy management, distribution of energy, and energy delivery (Antonopoulos et al., 2020; Al-Shetwi et al., 2020a; Ahmad et al., 2021). For instance, an optimal scheduling controller that uses AI optimization reduced the cost and emission in a micro-grid system that consists of different RE sources by $2.6 \%$ and $8.1 \%$, respectively (Abdolrasol et al., 2018) (targets 11.6 and 13.1). It is founded that AI utilization in RE can assist in achieving the future goals of the RE by developing accurate strategies in the control, simulation, decision, and optimization of RE systems. Toward achieving the SDGs, the use of AI in the RE based-power sector is now reaching emerging markets, where it may have a critical impact, as clean, cheap, and reliable energy is essential to development. The issues can be overcome over time by shifting knowledge of the power field to AI software firms. When properly designed, AI systems can indeed be useful in automating routine and organized operations, thereby helping people to address possible energy challenges in the future (Makala and Bakovic, 2020).

\section{Toward sustainable RE utilization}

As mentioned above, the accelerated deployment of RE has been motivated primarily by a wide variety of goals (drivers), including advancing economic growth, enhancing the security of energy and access to electricity and alleviating climate change (Lund, 2007). As explained in Fig. 2(a), the RE can achieve $100 \%$ of the SDG 7 target (Affordable and Clean Energy). However, toward sustainable RE utilization, the RE sources have to address some limitations and negative impact (illustrated in Fig. 2(b) based on the published evidence). At present, we have no access to modern energy for 13\% of the global population (Rehman, 2019). In the same context, based on the UN report (UN SDG, 2019), electricity is still unavailable for one in seven people. The bulk of this population lives in the world's developing rural areas (The World Bank, 2019). Therefore, the development of RE resources is theoretically enough to generate electricity and then fill the current energy gap. In addition, the utilization of these renewable sources can contribute toward achieving many targets of the 17 SDGs and provide multiple long-term benefits, including job creation, energy security, economic prospects, environmental development, and global warming prevention (Armin Razmjoo et al., 2020). However, no clear vision in this regard is presented across the world, especially in terms of economic support (SDG 8) to date. The reason may be because no noteworthy causal link is found between $\mathrm{RE}$ consumption and total factor productivity (TFP) growth (target 8.2). In contrast with non-renewables, energy consumption engenders have favorable externality that leads to economic growth (Tugcu and Tiwari, 2016). Furthermore, the integration of RE sources into the electrical grid still suffer from the existence of unique standards across the globe to regulate its connection efficiently toward a secure, reliable, and economic integration (Al-Shetwi et al., 2020b). Moreover, the RE strategic 
policy shall cover four key aims: security of energy, social equity, economic benefits, and protection of the environment (Liu et al., 2019). Some studies concluded that despite the advantages of biomass as RE source, its utilization might act as an inhibitor for the target (3.9) because it releases carbon monoxide, thereby leading to headache, dizziness, nausea, and premature death (Freiberg et al., 2018; Hamouda et al., 2020). In addition, biomass and other sources, such as solar farms, can affect the ecosystems (target 6.6) by cutting the wood for biomass energy operation and using the wetland for farm installation (Gasparatos et al., 2017; Hodges et al., 2019). Therefore, an urgent solution to these negative effects should be addressed. Furthermore, the marine RE could have negative environmental impacts with respect to SDG 14 (life below water), resulting from habitat loss, collision risks, noise, and electromagnetic fields of the RE devices (Inger et al., 2009). The marine RE can affect SDG 14 and SDG 15 through (a) increased underwater noise and collision risk (Caine, 2020); (b) during day-to-day operation, underwater noise, emission of electromagnetic fields and collision or avoidance with the energy structures represent other potential impacts on coastal species, particularly large predators (Suryakiran et al., 2020); (c) offshore wind energy and tidal energy may cause a small amount of acidification during the construction, operation and/or decommissioning (Gill, 2005); and (d) in spite of the fact that wind energy system is low-polluting, it involves spatial tension and can affect ecosystems, including fish, aquatic mammals, and birds (Hagos, 2007). Therefore, these negative impacts and limitations of RE should be addressed toward sustainable RE development and toward achieving more SDG targets.

\section{Conclusion}

The need for more urgent and intensive action to combat climate change and ensure sustainable growth is widely recognized. In support of this target, the development of the renewable energy (RE) industry has been increased dramatically, along with improving its efficiency using artificial intelligence (AI). However, their impact on achieving the sustainable development goals (SDGs) is not sufficiently covered. Therefore, this study discussed, analyzed, reviewed, and explored how the RE use including the AI applications can positively or negatively affect the achievement of the adopted 2030 agenda for sustainable development that includes 17 SDGs with their 169 targets. In this assessment, the 17 SDGs were divided into environment, society, and economy groups. These three groups are the key pillars of sustainable development. The method used to achieve the objectives of this study, an expert elicitation method-based consensus is used. The results have shown that the RE can positively affect the achievement of 75 targets (44.3\%) of all SDGs, but it may negatively impact the accomplishment of 27 targets, which represent $15.97 \%$ of all targets. The results have also shown that the AI application can positively help the RE achieve 42 targets (24.85\%). Within the three groups of SDGs, the development and utilization of RE can positively affect the achievement of $45 \%, 41 \%$, and $50 \%$ of the environment, society, and economic targets, respectively. On the other hand, it acts as an inhibitor toward achieving 37\%,14\%, and $10 \%$ of the environmental, economic, and society targets, respectively. In terms of using AI in RE applications, AI can positively affect the achievement of $23 \%, 28 \%$, and $22 \%$ of the environment, society, and economic targets, respectively. For the rest, no pieces of evidence are found. However, with the current development and exponential growth of RE share and $\mathrm{AI}$, along with addressing certain present limitations, these impacts may cover additional targets in the future toward sustainable development and clean energy.

\section{Code availability}

The analysis code and the examined cases that validated our method are available from the corresponding authors upon reasonable request.

\section{CRediT authorship contribution statement}

M.A. Hannan: Conceptualization, Funding acquisition, Investigation, Project administration, Supervision, Writing - review \& editing. Ali Q Al-Shetwi: Data curation, Formal analysis, Writing - original draft, Writing - review \& editing. Pin Jern Ker: Funding acquisition, Software, Supervision, Writing - review \& editing. R.A. Begum: Methodology. M. Mansor: Methodology, Writing review \& editing. S.A. Rahman: Software. Z.Y. Dong: Software, Visualization, Writing - review \& editing. S.K. Tiong: Software, Visualization, Writing - review \& editing. T.M. Indra Mahlia: Software, Validation, Writing - review \& editing. K.M. Muttaqi: Resources, Visualization, Writing - review \& editing.

\section{Declaration of competing interest}

The authors declare that they have no known competing financial interests or personal relationships that could have appeared to influence the work reported in this paper.

\section{Data availability}

The data that support the findings of this study are available from the corresponding authors upon reasonable request.

\section{Acknowledgments}

This work was supported by the LRGS project (Grant No. 20190101LRGS) from the Ministry of Higher Education, Malaysia under Universiti Tenaga Nasional, Malaysia and in part by the UNITEN Bold Refresh Publication Fund 2021, under Project J5100D4103.

\section{Appendix A. Supplementary data}

Supplementary material related to this article can be found online at https://doi.org/10.1016/j.egyr.2021.08.172.

\section{References}

Abdmouleh, Z., Alammari, R.A., Gastli, A., 2015. Review of policies encouraging renewable energy integration \& best practices. Renew. Sustain. Energy Rev. 45, 249-262.

Abdolrasol, M.G., Hannan, M.A., Mohamed, A., Amiruldin, U.A.U., Abidin, I.B.Z. Uddin, M.N., 2018. An optimal scheduling controller for virtual power plant and microgrid integration using the binary backtracking search algorithm. IEEE Trans. Ind. Appl. 54, 2834-2844.

Aceleanu, M.I., Serban, A.C., Burghelea, C., 2015. Greening the youth employment-A chance for sustainable development. Sustainability 7, 2623-2643.

Adefarati, T., Bansal R.C. Reliability, 2019. Economic and environmental analysis of a microgrid system in the presence of renewable energy resources. Appl. Energy 236, 1089-1114.

Adenle, A.A., 2020. Assessment of solar energy technologies in africaopportunities and challenges in meeting the 2030 agenda and sustainable development goals. Energy Policy 137 (2020), 111180.

Ahmad, T., Zhang, D., Huang, C., Zhang, H., Dai, N., Song, Y., et al., 2021. Artificial intelligence in sustainable energy industry: Status quo, challenges and opportunities. J. Cleaner Prod. 125834.

Al-faruq, U., Sagala, S., Rianawati, E., Currie, E., 2016. Assessment of Renewable Energy Impact to Community Resilience in Sumba Island. Resilience Development Initiative, p. 9.

Al-Hamamre, Z., Saidan, M., Hararah, M., Rawajfeh, K., Alkhasawneh, H.E., AlShannag, M., 2017. Wastes and biomass materials as sustainable-renewable energy resources for Jordan. Renew. Sustain. Energy Rev. 67, 295-314. 
Al-Shetwi, A.Q., Hannan, M., Jern, K.P., Alkahtani, A.A., Abas, A.P.G., 2020a. Power quality assessment of grid-connected PV system in compliance with the recent integration requirements. Electronics 9, 366.

Al-Shetwi, A.Q., Hannan, M., Jern, K.P., Mansur, M., Mahlia, T., 2020b. Gridconnected renewable energy sources: Review of the recent integration requirements and control methods. J. Cleaner Prod. 253, 119831.

Al-Waeli, A.H., Al-Kabi, A.H., Al-Mamari, A., Kazem, H.A., Chaichan, M.T., 2017. Evaluation of the economic and environmental aspects of using photovoltaic water pumping system. In: 9th International Conference on Robotic, Vision, Signal Processing and Power Applications. Springer, pp. 715-723.

Alipour, R., Alipour, R., Fardian, F., Koloor, S.S.R., Petrů, M., 2020a. Performance improvement of a new proposed Savonius hydrokinetic turbine: a numerical investigation. Energy Rep. 6, 3051-3066.

Alipour, R., Alipour, R., Rahimian Koloor, S.S., Petrů, M., Ghazanfari, S.A., 2020b. On the performance of small-scale horizontal axis tidal current turbines. Part 1: one single turbine. Sustainability 12, 5985.

Alvarez-Herranz, A., Balsalobre-Lorente, D., Shahbaz, M., Cantos, J.M., 2017. Energy innovation and renewable energy consumption in the correction of air pollution levels. Energy Policy 105, 386-397.

Amador, R., 2017. Rural renewable energy based infrastructure of the Philippines.

Antonopoulos, I., Robu, V., Couraud, B., Kirli, D., Norbu, S., Kiprakis, A., et al., 2020. Artificial intelligence and machine learning approaches to energy demand-side response: A systematic review. Renew. Sustain. Energy Rev. 130, 109899.

Appel, F., Ostermeyer-Wiethaup, A., Balmann, A., 2016. Effects of the German Renewable Energy Act on structural change in agriculture-The case of biogas. Utilities Policy 41, 172-182.

Armin Razmjoo, A., Sumper, A., Davarpanah, A., 2020. Energy sustainability analysis based on SDGs for developing countries. Energy Sources A 42, 1041-1056.

Atabi, F., 2004. Renewable energy in Iran: Challenges and opportunities for sustainable development. Int. J. Environ. Sci. Technol. 1, 69-80.

Ayodele, E., Misra, S., Damasevicius, R., Maskeliunas, R., 2019. Hybrid microgrid for microfinance institutions in rural areas-A field demonstration in West Africa. Sustain. Energy Technol. Assess. 35, 89-97.

Azimoh, C.L., Klintenberg, P., Mbohwa, C., Wallin, F., 2017. Replicability and scalability of mini-grid solution to rural electrification programs in sub-saharan africa. Renew. Energy 106, 222-231.

Bagheri, M., Shirzadi, N., Bazdar, E., Kennedy, C.A., 2018. Optimal planning of hybrid renewable energy infrastructure for urban sustainability: Green Vancouver. Renew. Sustain. Energy Rev. 95, 254-264.

Banos, R., Manzano-Agugliaro, F., Montoya, F., Gil, C., Alcayde, A., Gómez, J., 2011. Optimization methods applied to renewable and sustainable energy: A review. Renew. Sustain. Energy Rev. 15, 1753-1766.

Barron-Gafford, G.A., Pavao-Zuckerman, M.A., Minor, R.L., Sutter, L.F., BarnettMoreno, I., Blackett, D.T., et al., 2019. Agrivoltaics provide mutual benefits across the food-energy-water nexus in drylands. Nat. Sustain. 2, 848-855.

Batmunkh, S., Stennikov, V., Bat-Erdene, B., Erdenebaatar, A., 2018. Mongolia's potential in international cooperation in the Asian energy space. In: E3S Web of Conferences: EDP Sciences. p. 01006.

Behera, B.K., Rout, P.K., Behera, S., 2019. Water, energy and food security: Pillars for zero Hunger. In: Move Towards Zero Hunger. Springer, pp. 37-60.

Bertsiou, M., Feloni, E., Karpouzos, D., Baltas, E., 2018. Water management and electricity output of a hybrid renewable energy system (HRES) in fournoi island in Aegean Sea. Renew. Energy 118, 790-798.

Bonar, P.A., Bryden, I.G., Borthwick, A.G., 2015. Social and ecological impacts of marine energy development. Renew. Sustain. Energy Rev. 47, 486-495.

Bowen, T., del Ninno, C., Andrews, C., Coll-Black, S., Gentilini, U., Johnson, K., et al., 2020. International Energy Agency; International Renewable Energy Agency; United Nations Statistics Division; World Bank; World Health Organization. Policy..

Boza, P., Evgeniou, T., 2021. Artificial intelligence to support the integration of variable renewable energy sources to the power system. Appl. Energy 290, 116754.

Bryant, H.L., Lu, J., Richardson, J.W., Outlaw, J.L., 2010. Long-Term Effects of the US Renewable Fuel Standard on World Hunger.

Bugaje, I.M., 2006. Renewable energy for sustainable development in africa: a review. Renew. Sustain. Energy Rev. 10, 603-612.

Bukhary, S., Ahmad, S., Batista, J., 2018. Analyzing land and water requirements for solar deployment in the Southwestern United States. Renew. Sustain. Energy Rev. 82, 3288-3305.

Bulavskaya, T., Reynès, F., 2018. Job creation and economic impact of renewable energy in the Netherlands. Renew. Energy 119, 528-538.

Buonocore, J.J., Choma, E., Villavicencio, A.H., Spengler, J.D., Koehler, D.A., Evans, J.S., et al., 2019. Metrics for the sustainable development goals: renewable energy and transportation. Palgrave Commun. 5, 1-14.

Caine, C.A., 2020. The race to the water for offshore renewable energy: assessing cumulative and in-combination impacts for offshore renewable energy developments. J. Environ. Law 32, 83-109.

Callithen, N., Matthew, N., 2007. United Nations Development Programme (UNDP).
Carley, S., Davies, L.L., Spence, D.B., Zirogiannis, N., 2018. Empirical evaluation of the stringency and design of renewable portfolio standards. Nat. Energy 3, 754-763.

Carley, S., Konisky, D.M., 2020. The justice and equity implications of the clean energy transition. Nat. Energy 1-9.

Carroquino, J., Dufo-López, R., Bernal-Agustín, J.L., 2015. Sizing of off-grid renewable energy systems for drip irrigation in Mediterranean crops. Renew. Energy 76, 566-574.

Chandel, S., Shrivastva, R., Sharma, V., Ramasamy, P., 2016. Overview of the initiatives in renewable energy sector under the national action plan on climate change in India. Renew. Sustain. Energy Rev. 54, 866-873.

Chaouali, H., Salem, W.B., Mezghani, D., Mami, A., 2018. Fuzzy logic optimization of a centralized energy management strategy for a hybrid PV/PEMFC system feeding a water pumping station. Int. J. Renew. Energy Res. (IJRER) 8, 2190-2198

Chel, A., Kaushik, G., 2011. Renewable energy for sustainable agriculture. Agron. Sustain. Dev. 31, 91-118.

Chel, A., Kaushik, G., 2018. Renewable energy technologies for sustainable development of energy efficient building. Alexandria Eng. J. 57, 655-669.

Chen, C., Hu, Y., Marimuthu, K., Kumar, P.M., 2021. Artificial intelligence on economic evaluation of energy efficiency and renewable energy technologies. Sustain. Energy Technol. Assess. 47, 101358.

Clements, J.C., Chopin, T., 2017. Ocean acidification and marine aquaculture in north america: potential impacts and mitigation strategies. Rev. Aquac. 9, 326-341.

Cloke, J., Mohr, A., Brown, E., 2017. Imagining renewable energy: Towards a Social Energy Systems approach to community renewable energy projects in the Global South. Energy Res. Soc. Sci. 31, 263-272.

Collste, D., Pedercini, M., Cornell, S.E., 2017. Cornell SE policy coherence to achieve the SDGs: using integrated simulation models to assess effective policies. Sustain. Sci. 12, 921-931.

Colombo, E., Romeo, F., Mattarolo, L., Barbieri, J., Morazzo, M., 2018. An impact evaluation framework based on sustainable livelihoods for energy development projects: an application to ethiopia. Energy Res. Soc. Sci. 39, 78-92.

Cremiato, R., Mastellone, M.L., Tagliaferri, C., Zaccariello, L., Lettieri, P., 2018 Environmental impact of municipal solid waste management using Life Cycle Assessment: The effect of anaerobic digestion, materials recovery and secondary fuels production. Renew. Energy 124, 180-188.

Cunha, F.B.F., de Miranda Mousinho, M.C.A., Carvalho, L., Fernandes, F., Castro, C., Silva, M.S., et al., 2021. Renewable energy planning policy for the reduction of poverty in Brazil: lessons from Juazeiro. Environ. Dev. Sustain. 23, 9792-9810

Dalton, G., Lockington, D., Baldock, T., 2008. Feasibility analysis of standalone renewable energy supply options for a large hotel. Renew. Energy 33, 1475-1490.

Del Rio, P., Burguillo, M., 2009. An empirical analysis of the impact of renewable energy deployment on local sustainability. Renew. Sustain. Energy Rev. 13, 1314-1325.

DeNeve, J.-E., Sachs, J.D., 2020. The SDGs and human well-being: a global analysis of synergies, trade-offs, and regional differences. Sci. Rep. 10, 1-12.

Dhunny, A., Allam, Z., Lobine, D., Lollchund, M., 2019. Sustainable renewable energy planning and wind farming optimization from a biodiversity perspective. Energy 185, 1282-1297.

Diab, F., Lan, H., Ali, S., 2016. Novel comparison study between the hybrid renewable energy systems on land and on ship. Renew. Sustain. Energy Rev. 63, 452-463.

Diachuk, O., Chepeliev, M., Podolets, R., Trypolska, G., Venger, V., Saprykina, T., et al., 2018. Transition of Ukraine to the Renewable Energy by 2050. In Transition of Ukraine to the Renewable Energy By. p. 2050.

Ding, W., He, L., Zewudie, D., Zhang, H., Zafar, T.B., Liu, X., 2019. Gender and renewable energy study in Tibetan pastoral areas of China. Renew. Energy 133, 901-913.

Dóci, G., Gotchev, B., 2016. When energy policy meets community: Rethinking risk perceptions of renewable energy in Germany and the netherlands. Energy Res. Soc. Sci. 22, 26-35.

Drechsler, M., Egerer, J., Lange, M., Masurowski, F., Meyerhoff, J., Oehlmann, M. 2017a. Efficient and equitable spatial allocation of renewable power plants at the country scale. Nat. Energy 2, 1-9

Drechsler, M., Egerer, J., Lange, M., Masurowski, F., Meyerhoff, J., Oehlmann, M. 2017b. Efficient and equitable spatial allocation of renewable power plants at the country scale. Nat. Energy 2, 17124.

Ediger, VS., 2019. An integrated review and analysis of multi-energy transition from fossil fuels to renewables. Energy Procedia 156, 2-6.

Eitan, A., Herman, L., Fischhendler, I., Rosen, G., 2019. Community-private sector partnerships in renewable energy. Renew. Sustain. Energy Rev. 105, 95-104.

Elkadeem, M., Wang, S., Sharshir, S.W., Atia, E.G., 2019. Feasibility analysis and techno-economic design of grid-isolated hybrid renewable energy system for electrification of agriculture and irrigation area: A case study in Dongola, Sudan. Energy Convers. Manage. 196, 1453-1478. 
Essletzbichler, J., 2012. Renewable energy technology and path creation: A multi-scalar approach to energy transition in the UK. Eur. Plann. Stud. 20, 791-816.

Esteban, M., Portugal-Pereira, J., Mclellan, B.C., Bricker, J., Farzaneh, H., Djalilova, N., et al., 2018. 100\% renewable energy system in Japan: Smoothening and ancillary services. Appl. Energy 224, 698-707.

Fashina, A., Mundu, M., Akiyode, O., Abdullah, L., Sanni, D., Ounyesiga, L., 2019. The drivers and barriers of renewable energy applications and development in uganda: a review. Clean Technol. 1, 9-39.

Ferroukhi, R., Lopez-Peña, A., Kieffer, G., Nagpal, D., Hawila, D., Khalid, A., et al., 2016a. Renewable Energy Benefits: Measuring the Economics. International Renewable Energy Agency (IRENA), Abu Dhabi.

Ferroukhi, R., Lopez-Peña, A., Kieffer, G., Nagpal, D., Hawila, D., Khalid, A., et al., 2016b. Renewable energy benefits: measuring the economics. IRENA Int. Renew. Energy Agency 92.

Fraundorfer, M., Rabitz, F., 2020. The Brazilian renewable energy policy framework: Instrument design and coherence. Clim. Policy 20, 652-660.

Freiberg, A., Scharfe, J., Murta, V.C., Seidler, A., 2018. The use of biomass for electricity generation: A scoping review of health effects on humans in residential and occupational settings. Int. J. Environ. Res. Public Health 15, 354.

Freire-González, J., Puig-Ventosa, I., 2019. Reformulating taxes for an energy transition. Energy Econ. 78, 312-323.

Freire-Gormaly, M., 2018. Experimental characterization of membrane fouling under intermittent operation and its application to the optimization of solar photovoltaic powered reverse osmosis drinking water treatment systems.

Frid, C., Andonegi, E., Depestele, J., Judd, A., Rihan, D., Rogers, S.I., et al., 2012. The environmental interactions of tidal and wave energy generation devices. Environ. Impact Assess. Rev. 32, 133-139.

GA U, 2015. Transforming Our World: The 2030 Agenda for Sustainable Development. Division for Sustainable Development Goals, New York, NY, USA.

Gabriel, C.-A., Kirkwood, J., 2016. Business models for model businesses: Lessons from renewable energy entrepreneurs in developing countries. Energy Policy 95, 336-349.

Gasparatos, A., Doll, C.N., Esteban, M., Ahmed, A., Olang, T.A., 2017. Renewable energy and biodiversity: Implications for transitioning to a Green Economy. Renew. Sustain. Energy Rev. 70, 161-184.

Giday, Z.G., 2014. Technical and economic assessment of solar PV/diesel hybrid power system for rural school electrification in Ethiopia. Int. J. Renew. Energy Res. (IJRER) 3, 735-744.

Giddings, B., Hopwood, B., O'brien, G., 2002. Environment, economy and society: fitting them together into sustainable development. Sustain. Dev. 10, 187-196.

Gielen, D., Boshell, F., Saygin, D., Bazilian, M.D., Wagner, N., Gorini, R., 2019. The role of renewable energy in the global energy transformation. Energy Strategy Rev. 24, 38-50.

Gill, A.B., 2005. Offshore renewable energy: ecological implications of generating electricity in the coastal zone. J. Appl. Ecol. 605-615.

Global Status Report, 2021. Renewables 2021 Global Status Report-REN21.

Goel, S., Sharma, R., 2016. Feasibility study of hybrid energy system for off-grid rural water supply and sanitation system in Odisha, India. Int. J. Ambient Energy 37, 314-320.

Gökgöz, F., Güvercin, M.T., 2018. Energy security and renewable energy efficiency in EU. Renew. Sustain. Energy Rev. 96, 226-239.

Gontijo, J.C., Wagner, L.G., Souza, M.E.d., Possetti, G.R.C., 2018. Sanitation and drying of sewage sludge on radiant floors using solar energy and biogas: comparison between different thicknesses of deposited mass. Braz. Arch. Biol. Technol. 61.

Gove, B., Williams, L.J., Beresford, A.E., Roddis, P., Campbell, C., Teuten, E., et al., 2016. Reconciling biodiversity conservation and widespread deployment of renewable energy technologies in the UK. PLoS One 11, e0150956.

Grabara, J., Tleppayev, A., Dabylova, M., Mihardjo, L.W., Dacko-Pikiewicz, Z., 2021. Empirical research on the relationship amongst renewable energy consumption, economic growth and foreign direct investment in Kazakhstan and Uzbekistan. Energies 14, 332.

Griffith-Jones, S., Spratt, S., Andrade, R., Griffith-Jones, E., 2017. Investment in renewable energy, fossil fuel prices and policy implications for latin america and the caribbean.

Hagos, K.W., 2007. Impact of Offshore Wind Energy on Marine Fisheries in Rhode Island. White Paper in Integrated Coastal Science, RI Department of Environmental Management, Division of Fish and Wildlife.

Halabi, L.M., Mekhilef, S., 2018. Flexible hybrid renewable energy system design for a typical remote village located in tropical climate. J. Clean. Prod. 177, 908-924.

Halaweh, M., 2018. Artificial intelligence government (gov. 3.0): The UAE leading model. J. Artif. Intell. Res. 62, 269-272.

Halder, D., Mondal, S., Mukherjee, A., Ghosh, A., 2016. An overview on the treatment of wastewater using renewable energy. Int. J. Res. Eng. Technol. $5,78-83$.
Hamouda, R.A., El-Naggar, NE-A., Doleib, N.M., Saddiq, A.A., 2020. Bioprocessing strategies for cost-effective simultaneous removal of chromium and malachite green by marine alga enteromorpha intestinalis. Sci. Rep. 10, 1-19.

Hannan, M., Abdolrasol, M., Faisal, M., Ker, P.J., Begum, R., Hussain, A., 2019. Binary particle swarm optimization for cheduling MG integrated virtual power plant toward energy saving. IEEE Access 7, 107937-107951.

Hannan, M., Al-Shetwi, A.Q., Begum, R., Young, S., Hoque, M., Ker, P.J., et al., 2020a. The value of thermal management control strategies for battery energy storage in grid decarbonization: Issues and recommendations. J. Cleaner Prod. 124223.

Hannan, M., Ali, J.A., Lipu, M.H., Mohamed, A., Ker, P.J., Mahlia, T.I., et al., 2020b. Role of optimization algorithms based fuzzy controller in achieving induction motor performance enhancement. Nat. Commun. 11, 1-11.

Hannan, M., Begum, R., Al-Shetwi, A.Q., Ker, P., Mamun, M.Al., Hussain, A., et al. 2020c. Waste collection route optimisation model for linking cost saving and emission reduction to achieve sustainable development goals. Sustainable Cities Soc. 102393

Hannan, M.A., Lipu, M.H., Hussain, A., Ker, P.J., Mahlia, T., Mansor, M., et al., 2020d. Toward enhanced state of charge estimation of lithium-ion batteries using optimized machine learning techniques. Sci. Rep. 10, 1-15.

Hannan, M.A., Lipu, M.S.H., Hussain, A., Saad, M.H., Ayob, A., 2018. Neural network approach for estimating state of charge of lithium-ion battery using backtracking search algorithm. Ieee Access 6, 10069-10079.

Hannan, M., Tan, S.Y., Al-Shetwi, A.Q., Jern, K.P., Begum, R., 2020e. Optimized controller for renewable energy sources integration into microgrid: Functions, constraints and suggestions. J. Clean. Prod. 256, 120419.

Hansen, K., Mathiesen, B.V., Skov, I.R., 2019. Full energy system transition towards $100 \%$ renewable energy in Germany in 2050. Renew. Sustain. Energy Rev. 102, 1-13.

Harkouss, F., Fardoun, F., Biwole, P.H., 2019. Optimal design of renewable energy solution sets for net zero energy buildings. Energy 179, 1155-1175.

Hassanien, R.H.E., Li, M., Lin, W.D., 2016. Advanced applications of solar energy in agricultural greenhouses. Renew. Sustain. Energy Rev. 54, 989-1001.

Heidari, N., Pearce, J.M., 2016. A review of greenhouse gas emission liabilities as the value of renewable energy for mitigating lawsuits for climate change related damages. Renew. Sustain. Energy Rev. 55, 899-908.

Herweijer, C., Waughray, D., 2018. Fourth industrial revolution for the earth harnessing artificial intelligence for the earth. In: A Report of PricewaterhouseCoopers (PwC).

Hodges, D.G., Chapagain, B., Watcharaanantapong, P., Poudyal, N.C., Kline, K.L., Dale, V.H., 2019. Opportunities and attitudes of private forest landowners in supplying woody biomass for renewable energy. Renew. Sustain. Energy Rev. 113, 109205.

Hu, H., Xie, N., Fang, D., Zhang, X., 2018. The role of renewable energy consumption and commercial services trade in carbon dioxide reduction: Evidence from 25 developing countries. Appl. Energy 211, 1229-1244.

Hussein, E.A., 2017. Design of Renewable Energy System for a Mobile Office/Hospital in an Isolated Rural Area. Memorial University of Newfoundland.

Hymel, M., 2006. United states' experience with energy-based tax incentives: The evidence supporting tax incentives for renewable energy. Loy U Chi LJ. 38, 43.

Inger, R., Attrill, M.J., Bearhop, S., Broderick, A.C., Grecian, W.James., Hodgson, D.J., et al., 2009. Marine renewable energy: potential benefits to biodiversity? An urgent call for research. J. Appl. Ecol. 46, 1145-1153.

International Energy Agency, 2020. Renewables - global energy review 2020 analysis - IEA.

International Energy Agency (IEA), 2021. Renewables - Global Energy Review 2021 - Analysis - IEA.

IRENA, 2016. Renewable Energy Benefits: Measuring the Economics. International Renewable Energy Agency Abu Dhabi, United Arab Emirates.

Ivanovski, K., Hailemariam, A., Smyth, R., 2021. The effect of renewable and non-renewable energy consumption on economic growth: Non-parametric evidence. J. Clean. Prod. 286, 124956.

Jha, S.K., Bilalovic, J., Jha, A., Patel, N., Zhang, H., 2017. Renewable energy: Present research and future scope of Artificial Intelligence. Renew. Sustain. Energy Rev. 77, 297-317.

Jin, X., Wu, J., Mu, Y., Wang, M., Xu, X., Jia, H., 2017. Hierarchical microgrid energy management in an office building. Appl. Energy 208, 480-494.

Jones, N., 2018. How to stop data centres from gobbling up the world's electricity. Nature 561, 163-167.

Kalogirou, S., 2007. Artificial Intelligence in Energy and Renewable Energy Systems. Nova Publishers.

Kammen, D.M., Sunter, D.A., 2016. City-integrated renewable energy for urban sustainability. Science 352, 922-928.

Karmaker, A.K., Ahmed, M.R., Hossain, M.A., Sikder, M.M., 2018. Feasibility assessment \& design of hybrid renewable energy based electric vehicle charging station in Bangladesh. Sustain. Cities Soc. 39, 189-202.

Kaygusuz, K., 2012. Energy for sustainable development: A case of developing countries. Renew. Sustain. Energy Rev. 16, 1116-1126. 
Keith, A.E., French, J.J., 2019. Design and testing of a remote deployable water purification system powered by solar energy. Adv. Technol. Innov. 4, 30.

Khanna, N.Z., Zhou, N., Fridley, D., Ke, J., 2016. Quantifying the potential impacts of China's power-sector policies on coal input and $\mathrm{CO} 2$ emissions through 2050: A bottom-up perspective. Util. Policy 41 (2016), 128-138.

Kharchenko, V., Gusarov, V., Bolshev, V., 2019. Reliable electricity generation in RES-based microgrids. In: Handbook of Research on Smart Power System Operation and Control. IGI Global, pp. 162-187.

Khayyam, U., Nazar, U., 2021. Energy production and CO2 emissions: The case of coal fired power plants under China Pakistan economic corridor. J. Clean. Prod. 281, 124974.

Kim, K., Park, H., Kim, H., 2017. Real options analysis for renewable energy investment decisions in developing countries. Renew. Sustain. Energy Rev. 75, 918-926.

Kirsanova, N.Y., Lenkovets, O.M., Nikulina, A.Y., 2018. Renewable energy sources (RES) as a factor determining the social and economic development of the arctic zone of the Russian federation. Int. Multidiscip. Sci. GeoConf.: SGEM. 18, 679-686.

Konietzko, J., Bocken, N., Hultink, E.J., 2020. Circular ecosystem innovation: An initial set of principles. J. Clean. Prod. 253, 119942.

Korai, M.S., Mahar, R.B., Uqaili, M.A., 2016. Optimization of waste to energy routes through biochemical and thermochemical treatment options of municipal solid waste in Hyderabad, Pakistan. Energy Convers. Manage. 124, 333-343.

Kotrikla, A.M., Lilas, T., Nikitakos, N., 2017. Abatement of air pollution at an aegean island port utilizing shore side electricity and renewable energy. Mar. Policy 75, 238-248.

Kumar, M., 2020a. Social, Economic, and Environmental Impacts of Renewable Energy Resources. IntechOpen, Wind Solar Hybrid Renewable Energy System.

Kumar, M., 2020b. Social, economic, and environmental impacts of renewable energy resources. Wind Sol. Hybrid Renew. Energy Syst.

Kwasinski, A., Kwasinski, A., 2015. Increasing sustainability and resiliency of cellular network infrastructure by harvesting renewable energy. IEEE Commun. Mag. 53, 110-116.

Langhamer, O., 2012. Artificial reef effect in relation to offshore renewable energy conversion: state of the art. Sci. World J. 2012.

Le Guen, M., Mosca, L., Perera, A.T.D., Coccolo, S., Mohajeri, N., Scartezzini, J.-L., 2018. Improving the energy sustainability of a Swiss village through building renovation and renewable energy integration. Energy Build. 158, 906-923.

Li, X., Lin, R., Ni, G., Xu, N., Hu, X., Zhu, B., et al., 2018. Three-dimensional artificial transpiration for efficient solar waste-water treatment. Natl. Sci. Rev. 5, 70-77.

Lin, L., Yu, H., 2012. Offshore wave energy generation devices: impacts on ocean bio-environment. Acta Ecol. Sin. 32, 117-122.

Liu, W., Zhang, X., Feng, S., 2019. Does renewable energy policy work? Evidence from a panel data analysis. Renew. Energy 135, 635-642.

Lucas, H., Pinnington, S., Cabeza, L.F., 2018. Cabeza LF education and training gaps in the renewable energy sector. Sol. Energy 173, 449-455.

Lund, H., 2007. Renewable energy strategies for sustainable development. Energy 32, 912-919.

Ma, T., Yang, H., Lu, L., Peng, J., 2014. Technical feasibility study on a standalone hybrid solar-wind system with pumped hydro storage for a remote island in Hong Kong. Renew. Energy 69, 7-15.

Madriz-Vargas, R., Bruce, A., Watt, M., 2018. The future of community renewable energy for electricity access in rural central america. Energy Res. Soc. Sci. 35, $118-131$.

Makala, B., Bakovic, T., 2020. Artificial intelligence in the power sector.

Mandryk, O., Arkhypova, L., Pobigun, O., Maniuk, O., 2016. Renewable energy sources for sustainable tourism in the Carpathian region. In: IOP Conference Series: Materials Science and Engineering. IOP Publishing, 012007.

Manikandan, P., Karthick, S., Saravanan, S., Divya, T., 2018. Role of solar powered automatic traffic light controller for energy conservation. Int. Res. J. Eng. Technol. (IRJET) 5, 989-992.

Martin, G., Saikawa, E., 2017. Effectiveness of state climate and energy policies in reducing power-sector CO 2 emissions. Nat. Clim. Change 7, 912-919.

Martinot, E., Chaurey, A., Lew, D., Moreira, J.R., Wamukonya, N., 2002. Renewable energy markets in developing countries. Annu. Rev. Energy Environ. 27, 309-348.

Mauser, I., Müller, J., Allerding, F., Schmeck, H., 2016. Adaptive building energy management with multiple commodities and flexible evolutionary optimization. Renew. Energy 87, 911-921.

Miao, X., 2014. Renewables: Can harm environment. Nature 514, 168.

Mohammed, Y., Mustafa, M., Bashir, N., Ibrahem, I., 2017. Existing and recommended renewable and sustainable energy development in Nigeria based on autonomous energy and microgrid technologies. Renew. Sustain. Energy Rev. 75, 820-838.

Muh, E., Amara, S., Tabet, F., 2018. Sustainable energy policies in Cameroon: A holistic overview. Renew. Sustain. Energy Rev. 82, 3420-3429.

Münster, M., Meibom, P., 2011. Optimization of use of waste in the future energy system. Energy 36, 1612-1622.
Nabavi-Pelesaraei, A., Hosseinzadeh-Bandbafha, H., Qasemi-Kordkheili, P., Kouchaki-Penchah, H., Riahi-Dorcheh, F., 2016. Applying optimization techniques to improve of energy efficiency and GHG (greenhouse gas) emissions of wheat production. Energy 103, 672-678.

Nachrowi, N.D., 2012. The impact of Renewable Energy and GDP Per Capita on Carbon Dioxide emission in the G-20 Countries. Econ. Finance Indonesia 60, $145-174$.

Nazir, M.S., Mahdi, A.J., Bilal, M., Sohail, H.M., Ali, N., Iqbal, H.M., 2019. Environmental impact and pollution-related challenges of renewable wind energy paradigm-a review. Sci. Total Environ. 683, 436-444.

Nelson, S., Kuriakose, A.T., 2017. Gender and renewable energy: Entry points for women's livelihoods and employment. Clim. Invest. Funds.

Nerini, F.F., Sovacool, B., Hughes, N., Cozzi, L., Cosgrave, E., Howells, M., et al., 2019. Connecting climate action with other Sustainable Development Goals. Nat. Sustain. 2, 674-680.

Nerini, F.F., Tomei, J., To, L.S., Bisaga, I., Parikh, P., Black, M., et al., 2018. Mapping synergies and trade-offs between energy and the Sustainable Development Goals. Nat. Energy 3, 10-15.

Nie, S., Huang, Z., Huang, G., Yu, L., Liu, J., 2018. Optimization of electric power systems with cost minimization and environmental-impact mitigation under multiple uncertainties. Appl. Energy 221, 249-267.

Nissing, C., Von Blottnitz, H., 2010. Renewable energy for sustainable urban development: Redefining the concept of energisation. Energy Policy 38, 2179-2187.

Nizami, A., Shahzad, K., Rehan, M., Ouda, O., Khan, M., Ismail, I., et al., 2017. Developing waste biorefinery in makkah: a way forward to convert urban waste into renewable energy. Appl. Energy 186, 189-196.

Nižetić, S., Šolić, P., González-de, D.L.-d.-I., Patrono, L., 2020. Internet of things (IoT): Opportunities, issues and challenges towards a smart and sustainable future. J. Cleaner Prod. 274, 122877.

Oakleaf, J.R., Kennedy, C.M., Baruch-Mordo, S., Gerber, J.S., West, P.C., Johnson, J.A., et al., 2019. Mapping global development potential for renewable energy, fossil fuels, mining and agriculture sectors. Sci. Data 6, 1-17.

Okkonen, L., Lehtonen, O., 2016. Socio-economic impacts of community wind power projects in Northern Scotland. Renew. Energy 85, 826-833.

Olatomiwa, L., Blanchard, R., Mekhilef, S., Akinyele, D., 2018. Hybrid renewable energy supply for rural healthcare facilities: An approach to quality healthcare delivery. Sustain. Energy Technol. Assess. 30, 121-138.

Olatomiwa, L., Mekhilef, S., Ismail, M.S., Moghavvemi, M., 2016. Energy management strategies in hybrid renewable energy systems: A review. Renew. Sustain. Energy Rev. 62, 821-835.

Omri, A., Belaïd, F., 2021. Does renewable energy modulate the negative effect of environmental issues on the socio-economic welfare? J. Environ. Manag. 278,111483

Onifade, T.T., 2016. Hybrid renewable energy support policy in the power sector: The contracts for difference and capacity market case study. Energy Policy 95, 390-401.

Organization WH, 2014. Gender, Climate Change and Health. World Health Organization.

Owusu, P.A., Asumadu-Sarkodie, S., 2016. A review of renewable energy sources, sustainability issues and climate change mitigation. Cogent Eng. 3, 1167990.

Padilla, J.E., Hudson, A., 2019. United nations development programme (UNDP) perspectives on Asian large marine ecosystems. Deep Sea Res. II: Top. Stud. Oceanogr. 163, 127-129.

Petrevska, B., Cingoski, V., Serafimova, M., 2016. Sustainable tourism and hote management in Macedonia through the use of renewable energy sources. UTMS J. Econ. 7, 123-132.

Pfeiffer, B., Mulder, P., 2013. Explaining the diffusion of renewable energy technology in developing countries. Energy Econ. 40, 285-296.

Philibert, C., 2017. Renewable Energy for Industry. International Energy Agency, Paris.

Phuangpornpitak, N., Katejanekarn, T., 2016. Suitability analysis for implementing a renewable energy powered water purification system. Energy Procedia 89, 55-68.

Pino, M., Abarzúa, A.M., Astorga, G., Martel-Cea, A., Cossio-Montecinos, N., Navarro, R.X., et al., 2019. Sedimentary record from Patagonia, southern Chile supports cosmic-impact triggering of biomass burning, climate change, and megafaunal extinctions at $12.8 \mathrm{ka}$. Sci. Rep. 9, 1-27.

Poggi, F., Firmino, A., Amado, M., 2018. Planning renewable energy in rural areas: Impacts on occupation and land use. Energy 155, 630-640.

Ragosa, G., Warren, P., 2019. Unpacking the determinants of cross-border private investment in renewable energy in developing countries. J. Cleaner Prod. 235, 854-865.

Rahbar, K., Chai, C.C., Zhang, R., 2016. Energy cooperation optimization in microgrids with renewable energy integration. IEEE Trans. Smart Grid 9, $1482-1493$.

Rahman, F.A., Aziz, M.M.A., Saidur, R., Bakar, W.A.W.A, Hainin, M., Putrajaya, R., et al., 2017. Pollution to solution: Capture and sequestration of carbon dioxide (CO2) and its utilization as a renewable energy source for a sustainable future. Renew. Sustain. Energy Rev. 71, 112-126. 
Ram, M., Aghahosseini, A., Breyer, C., 2020. Job creation during the global energy transition towards $100 \%$ renewable power system by 2050 . Technol. Forecast. Soc. Change 151, 119682.

Ramakumar, R., Hughes, W.L., 1981. Renewable energy sources and rural development in developing countries. IEEE Trans. Educ. 24, 242-251.

Ramchurn, S.D., Vytelingum, P., Rogers, A., Jennings, N.R., 2012. Putting the'smarts' into the smart grid: a grand challenge for artificial intelligence. Commun. ACM 55, 86-97.

Re-thinking 2050, 2015. A 100\% renewable energy vision for European Union. In: European Renewable Energy Council.

Rehman, A., 2019. The nexus of electricity access, population growth, economic growth in Pakistan and projection through 2040. Int. J. Energy Sect. Manag..

Robles, E., Haro-Larrode, M., Santos-Mugica, M., Etxegarai, A., Tedeschi, E., 2019. Comparative analysis of European grid codes relevant to offshore renewable energy installations. Renew. Sustain. Energy Rev. 102, 171-185.

Saidi, K., Mbarek, M.B., 2016. Nuclear energy, renewable energy, CO2 emissions, and economic growth for nine developed countries: Evidence from panel Granger causality tests. Prog. Nucl. Energy 88, 364-374.

Salim, H.K., Padfield, R., Hansen, S.B., Mohamad, S.E., Yuzir, A., Syayuti, K., et al., 2018. Global trends in environmental management system and ISO14001 research. J. Cleaner Prod. 170, 645-653.

Savelyeva, T., Douglas, W., 2017. Global consciousness and pillars of sustainable development. Int. J. Sustain. Higher Educ..

Schuster, E., Bulling, L., Köppel, J., 2015. Consolidating the state of knowledge: a synoptical review of wind energy's wildlife effects. Environ. Manag. 56, 300-331.

Schwerhoff, G., Sy, M., 2017. Financing renewable energy in Africa-Key challenge of the sustainable development goals. Renew. Sustain. Energy Rev. 75, 393-401.

Semeraro, T., Pomes, A., Del Giudice, C., Negro, D., Aretano, R., 2018. Planning ground based utility scale solar energy as green infrastructure to enhance ecosystem services. Energy Policy 117, 218-227.

Sharma, P., 2018. Role of rural electrification and renewable energy on poverty in India: A state wise analysis. Asia Pac. J. Multidiscip. Res. 6.

Shaukat, N., Khan, B., Ali, S., Mehmood, C., Khan, J., Farid, U., et al., 2018. A survey on electric vehicle transportation within smart grid system. Renew. Sustain. Energy Rev. 81, 1329-1349.

Shen, W., Chen, X., Qiu, J., Hayward, J.A., Sayeef, S., Osman, P., et al., 2020. A comprehensive review of variable renewable energy levelized cost of electricity. Renew. Sustain. Energy Rev. 133, 110301.

Shields, M.A., Woolf, D.K., Grist, E.P., Kerr, S.A., Jackson, A.C., Harris, R.E., et al., 2011. Marine renewable energy: The ecological implications of altering the hydrodynamics of the marine environment. Ocean Coast. Manag. 54, 2-9.

Shirley, R., Lee, C-J., Njoroge, H.N., Odera, S., Mwanzia, P.K., Malo, I., et al., 2019. Powering jobs: The employment footprint of decentralized renewable energy technologies in sub saharan africa. J. Sustain. Res. 2.

Simon, C.A., 2020. Alternative Energy: Political, Economic, and Social Feasibility. Rowman \& Littlefield Publishers.

Simsek, H.A., Simsek, N., 2013. Recent incentives for renewable energy in Turkey. Energy Policy 63, 521-530.

Situmorang, Y.A., Zhao, Z., Yoshida, A., Abudula, A., Guan, G., 2020. Small-scale biomass gasification systems for power generation ( $<200 \mathrm{~kW}$ class): A review. Renew. Sustain. Energy Rev. 117, 109486.

Solaun, K., Cerdá, E., 2019. Climate change impacts on renewable energy generation. A review of quantitative projections. Renew. Sustain. Energy Rev. 116, 109415.

Sovacool, B.K., 2013. Expanding renewable energy access with pro-poor public private partnerships in the developing world. Energy Strategy Rev. 1, 181-192.

Srivastava, S.K., 2020. Application of artificial intelligence in renewable energy. In: 2020 International Conference on Computational Performance Evaluation (ComPE). IEEE, pp. 327-331.

Subiyanto, S., Mohamed, A., Hannan, M., 2012. Intelligent maximum power point tracking for PV system using hopfield neural network optimized fuzzy logic controller. Energy Build. 51, 29-38.

Subramanian, M., 2014. Deadly dinners. Nature 509, 548.

Surana, K., Anadon, L.D., 2015. Public policy and financial resource mobilization for wind energy in developing countries: a comparison of approaches and outcomes in China and India. Global Environ. Change 35, 340-359.

Suryakiran, M.N.S., Begum, W., Sudhakar, R., Tiwari, S.K., 2020. Development of wind energy technologies and their impact on environment: A review. Adv. Smart Grid Technol. 51-62.
Swain, R.B., Karimu, A., 2020. Renewable electricity and sustainable development goals in the EU. World Dev. 125, 104693.

Tabanjat, A., Becherif, M., Hissel, D., Ramadan, H.S., 2018. Energy management hypothesis for hybrid power system of H2/WT/PV/GMT via AI techniques. Int. J. Hydrogen Energy 43, 3527-3541.

The World Bank, 2019. Access to electricity (\% of population).

Thiam D.R. Renewable energy, 2011. Poverty alleviation and developing nations: Evidence from Senegal. J. Energy South. Afr. 22, 23-34.

Tugcu, C.T., Tiwari, A.K., 2016. Does renewable and/or non-renewable energy consumption matter for total factor productivity (TFP) growth? Evidence from the BRICS. Renew. Sustain. Energy Rev. 65, 610-616.

Turney, D., Fthenakis, V., 2011. Environmental impacts from the installation and operation of large-scale solar power plants. Renew. Sustain. Energy Rev. 15, 3261-3270.

UN SDG, 2019. Sustainable development goal 7 | SDG7 affordable and clean energy.

United Nations Educational, S., Organization, C., 2020. Global education monitoring report 2020: Inclusion and education: All means all.

United Nations Human Rights, 2016. Sustainable Development Goals Related Human Rights. United Nations Human Rights, 2016.

Vinuesa, R., Azizpour, H., Leite, I., Balaam, M., Dignum, V., Domisch, S., et al., 2020. The role of artificial intelligence in achieving the Sustainable Development Goals. Nature Commun. 11, 1-10.

Vyhmeister, E., Muñoz, C.A., Miquel, J.M.B., Moya, J.P., Guerra, C.F., Mayor, L.R. et al., 2017. A combined photovoltaic and novel renewable energy system: An optimized techno-economic analysis for mining industry applications. J. Cleaner Prod. 149, 999-1010.

Wagh, M., Kulkarni, V., 2018. Modeling and optimization of integration of Renewable Energy Resources (RER) for minimum energy cost, minimum $\mathrm{CO} 2$ emissions and sustainable development, in recent years: A review. Mater. Today: Proc. 5, 11-21.

Walmsley, M.R., Walmsley, T.G., Atkins, M.J., Kamp, P.J., Neale, J.R., 2014. Minimising carbon emissions and energy expended for electricity generation in new zealand through to 2050. Appl. Energy 135, 656-665.

Wang, R., Hsu, S.-C., Zheng, S., Chen, J.-H., Li, X.I., 2020. Renewable energy microgrids: Economic evaluation and decision making for government policies to contribute to affordable and clean energy. Appl. Energy 274, 115287.

Wang, X., Palazoglu, A., El-Farra, N.H., 2015. Operational optimization and demand response of hybrid renewable energy systems. Appl. Energy 143, 324-335.

Wei, M., Patadia, S., Kammen, D.M., 2010. Putting renewables and energy efficiency to work: How many jobs can the clean energy industry generate in the US? Energy Policy 38, 919-931.

Willsteed, E., Gill, A.B., Birchenough, S.N., Jude, S., 2017. Assessing the cumulative environmental effects of marine renewable energy developments: Establishing common ground. Sci. Total Environ. 577, 19-32.

Wright, A., 2019. Regulating offshore energy: Europe as a model for regulation. NCJ Int'L Lett. 45, 69.

Yang, J., Liu, H., Wang, Y., Qiu, Z., Dong, Z., Suzuki, T., et al., 0000. Key index framework for quantitative sustainability assessment of energy infrastructures in a smart city: An example of Western Sydney. Energy Convers. Econ.

Yang, T., Zhao, L., Wang, C., 2019. Review on application of artificial intelligence in power system and integrated energy system. Autom. Electr. Power Syst. 43, 2-14.

Zahnd, A., Kimber, H.M., 2009. Benefits from a renewable energy village electrification system. Renew. Energy 34, 362-368.

Zahraee, S., Assadi, M.K., Saidur, R., 2016. Application of artificial intelligence methods for hybrid energy system optimization. Renew. Sustain. Energy Rev. $66,617-630$.

Zamfir, A., 2012. Implementing regional renewable energy projects through public-private partnerships. Bus. Excell. Manag. 2, 77-84.

Zhu, Y., Wang, Z., Yang, J., Zhu, L., 2020. Does renewable energy technological innovation control China's air pollution? A spatial analysis. J. Cleaner Prod. 250, 119515.

Zvezdov, I.M., 2020. The EU legal and regulatory framework for measuring damage risks to the biodiversity of the marine environment. Environ. Policy: Econ. Perspect. 121-137. 